\title{
Long Noncoding RNA LINC01133 Confers Tumor-Suppressive Functions in Ovarian Cancer by Regulating Leucine-Rich Repeat Kinase 2 as an miR-205 Sponge
}

\author{
Min Liu, ${ }^{*}$ Cuiping Shen, ${ }^{\dagger}$ and Changxiu Wang
}

From the Departments of Reproductive Medicine* and Obstetrics and Gynecology, ${ }^{\ddagger}$ Linyi People’s Hospital, Linyi; and the Department of Obstetrics and Gynecology, ${ }^{\dagger}$ Rizhao People’s Hospital, Rizhao, People's Republic of China

\author{
Accepted for publication \\ July 26, 2019. \\ Address correspondence to \\ Changxiu Wang, M.M., \\ Department of Obstetrics and \\ Gynecology, Linyi People's \\ Hospital, No. 27, Jiefang Rd., \\ Lanshan District, Linyi 276000 , \\ Shandong Province, PR Chi- \\ na. E-mail: wangaxiu1981@ \\ 163.com.
}

\begin{abstract}
Ovarian cancer represents one of the most commonly occurring malignant tumors among females. Long noncoding RNAs act as biomarkers associated with the pathophysiology of multiple kinds of malignancies, including ovarian cancer. This study aimed to clarify the molecular mechanism of LINC01133 in the progression of ovarian cancer. Initially, microarray-based analysis was used to screen differentially expressed long noncoding RNAs and miRNAs, with LINC01133 and miR-205 obtained for this study. The biological functions of LINC01133, miR-205, and leucine-rich repeat kinase 2 (LRRK2) were validated on cell proliferation, migration, and invasion of ovarian cancer through gain-of-function and loss-offunction experiments. Finally, tumorigenesis was measured in vivo by inducing tumor xenograft in nude mice. The findings revealed a poor expression of LINC01133 in ovarian cancer tissue and cell, which was predominantly expressed in the cytoplasm. LINC01133 repressed cell proliferation, invasion, migration, and tumorigenic ability. miR-205 targeted and negatively regulated LRRK2. LINC01133 was also found to function as an miR-205 sponge to decrease ovarian cancer cell proliferation, migration, and invasion by elevating LRRK2. These in vitro findings were reproduced in vivo on tumor xenograft in nude mice. In conclusion, because of its role as an miR-205 sponge, LINC01133 repressed the development of ovarian cancer by up-regulating LRRK2. (Am J Pathol 2019, 189: 2323-2339; https:// doi.org/10.1016/j.ajpath.2019.07.020)
\end{abstract}

Ovarian cancer is the seventh most prevalent cancer in females worldwide, accounting for approximately $4 \%$ of all of the new cases of cancer in females. ${ }^{1}$ According to the latest data, there are 22,240 newly diagnosed ovarian cancer cases and there were 14,070 deaths in $2018 .^{2}$ There are several factors that have been identified that can induce ovarian cancer in females, including the early or late onset of menarche and the number of lifelong menstrual cycles. ${ }^{3}$ Ovarian cancer is mostly diagnosed in the advanced stages because of the lack of screening examinations that detect the disease at an early stage and the asymptomatic nature of the disease during the early stage. ${ }^{4}$ Although the first-line chemotherapy is effective for approximately $80 \%$ of ovarian cancer patients, $>70 \%$ of patients with advanced stage ovarian cancer show recurrence. ${ }^{5}$ There has been an increase in the studies that are conducted with the main focus of understanding the role of long noncoding RNAs (lncRNAs) in tumorigenesis of ovarian cancer. ${ }^{6}$

LncRNAs, >200 nucleotides, can regulate gene expression at both the posttranscriptional and transcriptional levels. ${ }^{7}$ LncRNAs can play a functional role as competitive endogenous RNAs and participate in different biological processes and tumorigenesis ${ }^{8}$ LINC01133 represses the expression of miR-106a-3p because of the role as a competitive endogenous RNA of miR-106a-3p and, thus, suppresses the expression of adenomatous polyposis coli. The LINC01133/miR-106 a-3p axis represses epithelialmesenchymal transition and metastasis of gastric cancer

Disclosures: None declared. 
cells via the inactivation of the $\mathrm{Wnt} / \beta$-catenin pathway in an adenomatous polyposis coli-dependent manner. In addition, by binding to SRSF6, LINC01133 can inhibit epithelial-mesenchymal transition and migration in colorectal cancer. ${ }^{10}$ The noncoding miRNAs have been implicated in the occurrence and development of ovarian cancer. ${ }^{11}$ miR-205 accelerates cell proliferation and invasion of ovarian cancer cells. ${ }^{12}$ In this study, using luciferase activity assay, leucine-rich repeat kinase 2 (LRRK2) was identified as a target of miR-205. LRRK2 has a mechanistic link with innate immunity and is implicated in the inflammatory response. ${ }^{13}$ This study investigated the regulatory relationships between LINC01133 and its downstream miRNAs and proteins, along with the underlying mechanism of LINC01133 in modulating ovarian cancer progression.

\section{Materials and Methods}

\section{Ethics Statement}

Before this study, written informed consents were obtained from all participants. The protocols of this study were approved by the Ethics Committee of Linyi People's Hospital (Linyi, China) and based on the ethical principles for medical research involving human subjects of the Declaration of Helsinki. Animal experiments were performed in strict accordance with the NIH's Guide for the Care and Use of Laboratory Animals. ${ }^{14}$ The protocol of animal experiments was approved by the Institutional Animal Care and Use Committee of Linyi People's Hospital.

\section{Microarray-Based Analysis}

Ovarian cancer-related gene expression data sets were obtained from the Gene Expression Omnibus database (https://www.ncbi.nlm.nih.gov/geo, last accessed February 2019). Differentially expressed lncRNA, gene, and miRNA were screened out from data sets GSE14407, GSE38666, and GSE83693, respectively. The background correction and standardization preprocessing were performed using the affy package in $\mathrm{R}$ language software version 3.5.1, ${ }^{15}$ and the limma package was employed to identify differentially expressed miRNAs or genes with $\mid \log 2$ fold change $\mid>2.0$ and adj.P.Val (the corrected $P$ value $)<0.05$ used as the screening threshold, ${ }^{16}$ after which the heat maps of differentially expressed miRNAs or genes were drawn. The subcellular localization of IncRNA was predicted with the use of the IncATLAS database (http://ncatlas.crg.eu, last accessed February 2019). The downstream regulatory miRNA of IncRNA and the downstream regulatory target gene of miRNA were predicted by the RNA22 database (https://cm. jefferson.edu/rna22/interactive, last accessed February 2019). The predicted results were compared with differentially expressed miRNAs by jvenn for screening
Table 1 The Primer Sequences for Real-Time Quantitative RT-PCR

\begin{tabular}{ll}
\hline Target & Primer sequences \\
\hline miR-205 & F: 5'-CCTTCATTCCACCGGAGT-3' \\
& R: 5'-GAACTTCACTCCACTGAAATCTG-3' \\
Leucine-rich & F: 5'-GAAAGGCCTACTTCTGCCCA-3' \\
repeat kinase 2 & R: 5'-GATGTGTAGCAACCATGCATTCA-3' \\
LINC01133 & F: 5'-TAGCTTCCCTTTGGATCTGG-3' \\
& R: 5'-GGAGCCTCGTTGTTAATGG-3' \\
U6 small & F: 5'-GCTTCGGCAGCACATATACTAAAAT-3' \\
nuclear RNA & R: 5'-CGCTTCACGAATTTGCGTGTCAT-3' \\
Cyclin D1 & F: 5'-ACCTGGATGCTGGAGGTCT-3' \\
& R: 5'-GCTCCATTTGCAGCAGCTC-3' \\
Cyclin D3 & F: 5'-CGTGGTCGGTGTAGATGC-3' \\
& R: 5'-TGGATGCTGGAGGTATGTG-3' \\
Cyclin dependent & F: 5'-GGTCCTCCACCGAGACCTTA-3' \\
kinase 2 & R: 5'-GAAAGATCCGGAAGAGCTGG-3' \\
Vimentin & F: 5'-CGCCAGATGCGTGAAATGG-3' \\
& R: 5'-ACCAGAGGGAGTGAATCCAGA-3' \\
N-cadherin & F: 5'-CAACTTGCCAGAAAACTCCAGG-3' \\
E-cadherin & R: 5'-ATGAAACCGGGCTATCTGCTC-3' \\
Flyceraldehyde- & F: 5: 5'-ATGGACCACGTACAAGGGTC-3' \\
3-phosphate & R: 5'-AAGTTGTCATGGATGACCTTG-3' \\
dehydrogenase & \\
\hline
\end{tabular}

$F$, forward; $R$, reverse.

miRNA. The prediction for target genes of the differentially expressed miRNA was performed by the online software on the websites miRDB (http://www. mirdb.org, last accessed February 2019), DIANA (http:// diana.imis.athena-innovation.gr/DianaTools/index.php? $r=$ microT_CDS/index, last accessed February 2019), TargetScan, mirDIP (http://ophid.utoronto.ca/mirDIP, last accessed February 2019) and RNA22. Those target genes were intersected using the jvenn tool.

\section{Study Subjects}

On the basis of the Revised International Federation of Gynecology and Obstetrics system, ${ }^{17} 50$ cases of ovarian cancer tissues were obtained from patients diagnosed with ovarian cancer, who had received tumor resection in Linyi People's Hospital between May 2015 and May 2016. In addition, 30 normal ovarian tissues were collected from women who had undergone a hysterectomy for benign tumor but had not experienced local or systemic therapy before operation in Linyi People's Hospital from December 2015 to December 2016. All specimens were immediately stored after being obtained in liquid nitrogen at $-80^{\circ} \mathrm{C}$. The specimens had complete clinical data and were assessed by histopathology.

\section{Culturing of Cells}

The normal ovarian epithelial cell line IOSE80 and ovarian cancer epithelial cell lines SKOV-3, HO-8910, and 

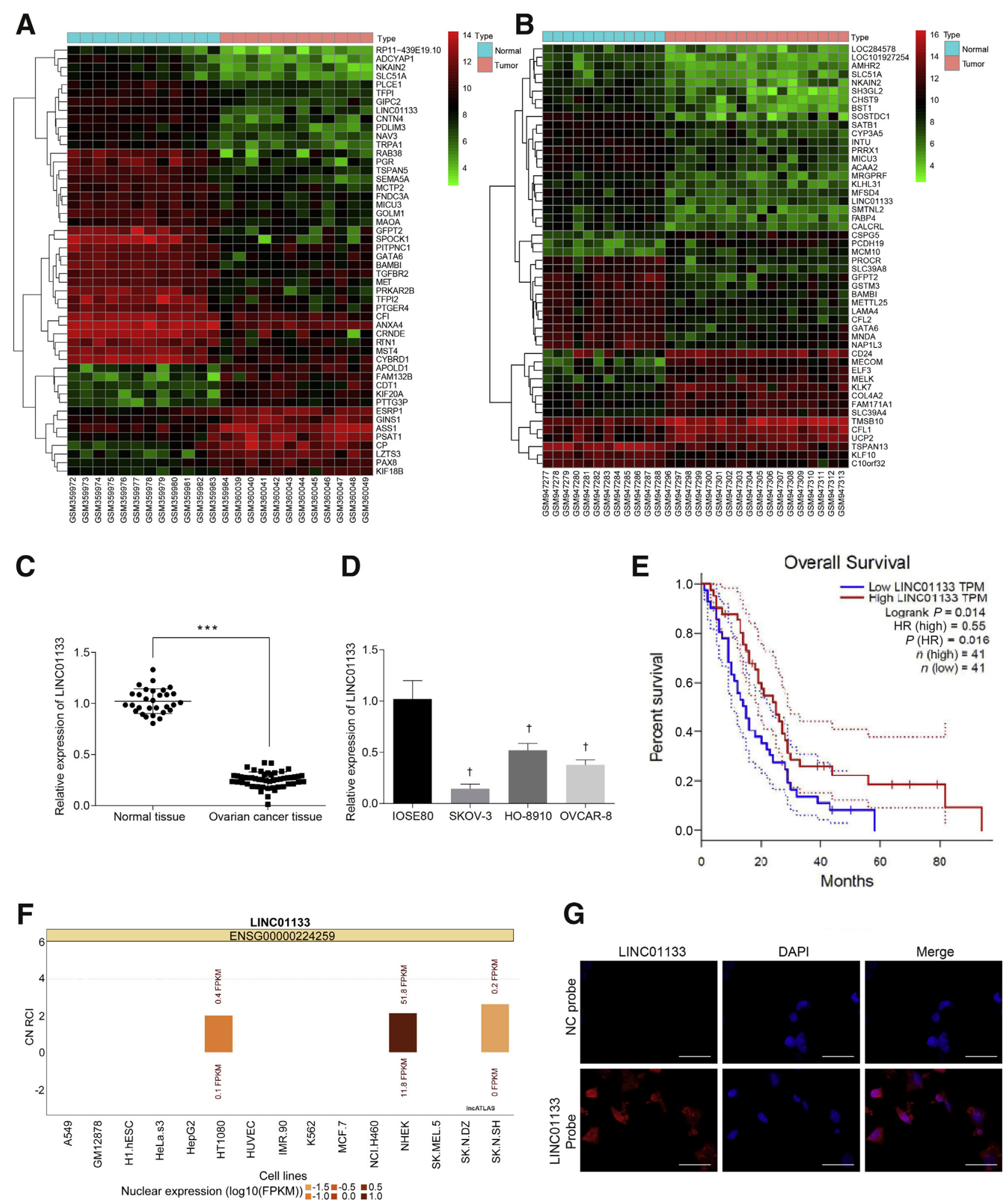

G
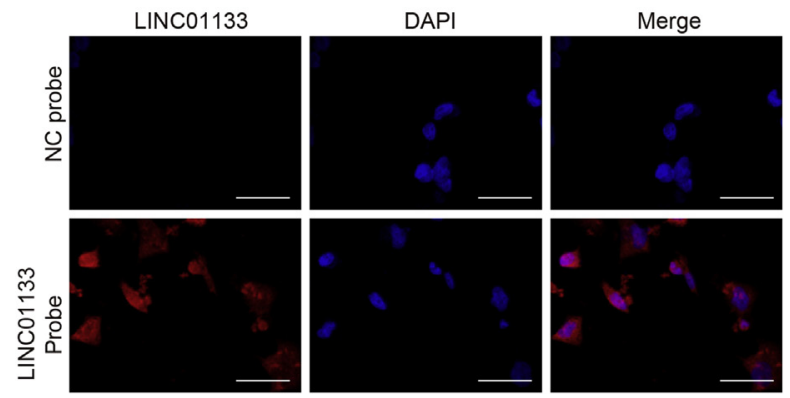

Figure 1 LINC01133 is down-regulated in ovarian cancer tissues and cells. A and B: The heat map of the first 50 differentially expressed genes in GSE14407 (A) and GSE38666 (B) gene expression data sets. C: The expression of LINC01133 in the ovarian cancer tissues and normal ovarian tissues. D: The expression of LINC01133 in IOSE80, SKOV-3, H0-8910, and OVCAR-8 cell lines. E: The overall survival of ovarian cancer patients with high or low expression of LINC01133. F: The prediction of subcellular localization of LINC01133 on long noncoding RNA ATLAS. G: The distribution of LINC01133 in cells detected by RNA fluorescence in situ hybridization. Unpaired $t$-test was used for data analysis between the two groups. One-way analysis of variance was used for comparisons among multiple groups with Tukey's post hoc test conducted. The experiment was repeated three times. Measurement data are expressed as means \pm SD (D). $n=50$ ovarian cancer tissue (C); $n=30$ normal ovarian tissues (C). ${ }^{* * *} P<0.001$ versus normal ovarian tissues; ${ }^{\dagger} P<0.05$ versus IOSE80 cell line. Scale bars $=50$ $\mu \mathrm{m}(\mathbf{G})$. C, cytoplasmic; HR, hazard ratio; N, nuclear; RCI, relative concentration index. 


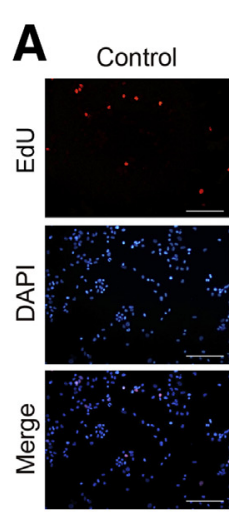

B
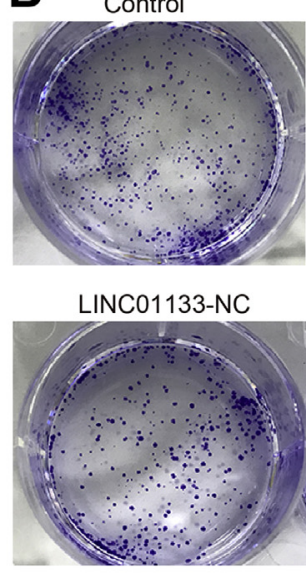

C control

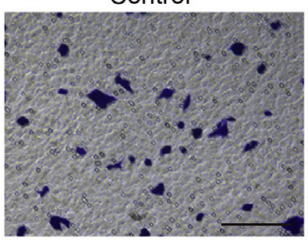

LINC01133-NC

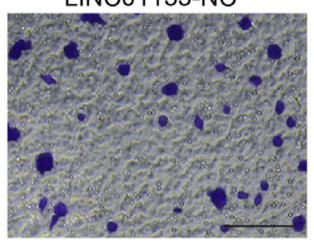

D Control

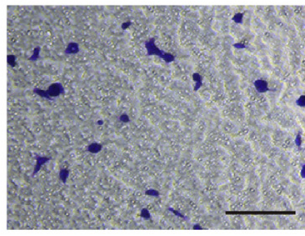

LINC01133-NC

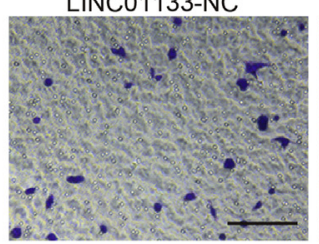

sh-LINC01133-NC
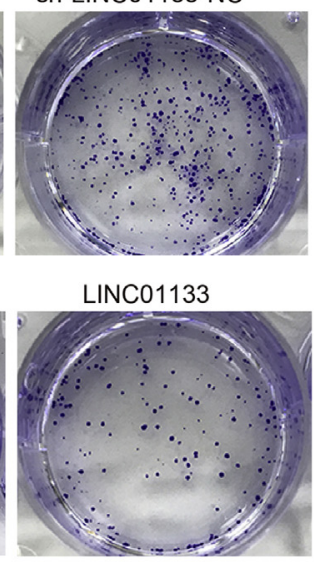

sh-LINC01133-NC

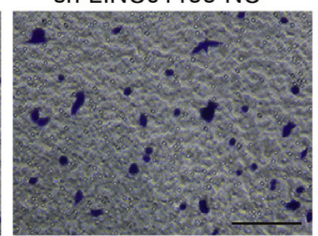

LINC01133

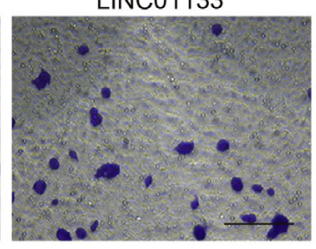

sh-LINC01133
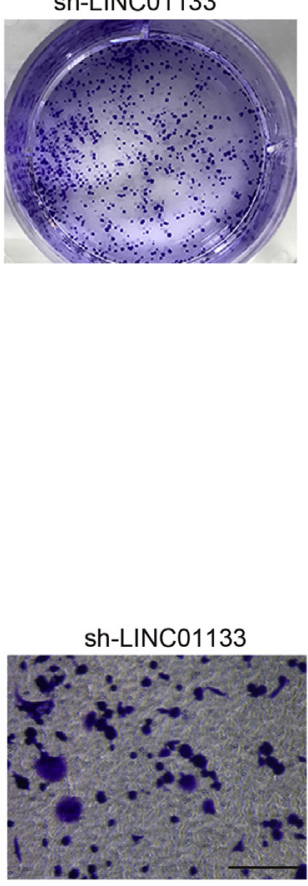$$
\text { 흔 }
$$

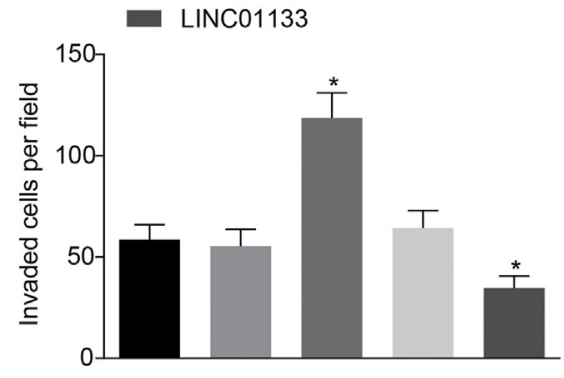

Control

sh-LINC01133-NC

- sh-LINC01133

LINC01133-NC

LINC01133

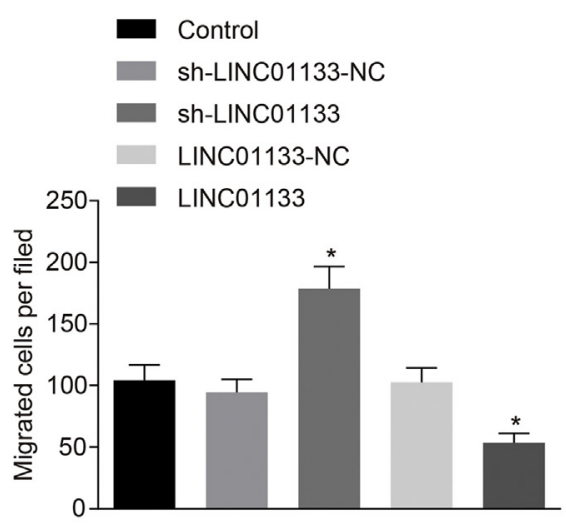


OVCAR-8, procured from ATCC (Manassas, VA), were adopted in this study. The cells were cultured in RPMI 1640 medium supplemented with $10 \%$ fresh serum, $100 \mathrm{U} / \mathrm{mL}$ penicillin, and $100 \mathrm{U} / \mathrm{mL}$ streptomycin with $5 \% \mathrm{CO}_{2}$ at $37^{\circ} \mathrm{C}$, with the medium renewed every 2 days. When cell confluence reached $80 \%$ to $90 \%$, the cells grown in the exponential phase were harvested for subsequent experiments.

\section{Plasmid Construction and Cell Treatment}

The sequences of LINC01133, miR-205, and LRRK2 were retrieved from the National Center of Biotechnology Information. On the basis of the pCMV-Flag-N/C vector (Shanghai Sangon Biotechnology Co, Ltd, Shanghai, China), the plasmids used in the study were constructed, which included miR-205 mimic, miR-205 mimic-negative control (NC), short hairpin RNA (sh)-LRRK2, shLRRK2-NC, LRRK2, LRRK2-NC, sh-LINC01133, shLINC01133-NC, LINC01133, and LINC01133-NC plasmids. The cells at the third passage were detached and plated into a 24-well plate. When the single layer of cells was formed, the culture medium was discarded. Subsequently, the cells were grouped after transfection on the basis of the protocols of Lipofectamine 2000 (Invitrogen, Carlsbad, CA).

\section{RNA Isolation and Quantification}

TRIzol reagent (Takara Inc., Dalian, China) was employed for the extraction of total RNA. Next, RNA and miRNA were reverse transcribed into cDNA with the use of the PrimeScript RT reagent Kit (Takara Inc.) and One Step PrimeScript miRNA cDNA Synthesis Kit (Takara Inc.), respectively. With glyceraldehyde-3-phosphate dehydrogenase $(G A P D H)$ regarded as the housekeeping gene for genes, and $U 6$ for miR-205, the cDNA was subjected to real-time quantitative RT-PCR (RT-qPCR) using Quanti-Tect SYBR Green PCR kit. Subsequent experiments were performed using SYBR Premix Ex Taq II kit (Takara, Tokyo, Japan). All primers are shown in Table 1. RT-qPCR was performed on the ABI 7500 quantitative PCR instrument (Applied Biosystems, Foster City, CA). The $\mathrm{Ct}$ values were recorded, with the $2^{-\Delta \Delta \mathrm{Ct}}$ method applied to quantify the relative expression.

\section{Western Blot Analysis}

The total proteins from cells were extracted, with protein separated using SDS-PAGE, followed by the transfer of the protein onto a polyvinylidene difluoride membrane. After being blocked by $5 \%$ skim milk, the membrane was incubated at $4^{\circ} \mathrm{C}$ overnight with the diluted primary rabbit antibodies: cyclin D1 (dilution 1:10,000; ab134175), cyclin D3 (dilution 1:100; ab183338), cyclin-dependent kinase 2 (CDK2; dilution 1:1000; ab32147), vimentin (dilution 1:1000; ab92547), $\mathrm{N}$-cadherin (dilution 1:1000; ab18203), E-cadherin (dilution 1:10,000; ab40772), and GAPDH (dilution 1:2500; ab9485). Subsequently, the membrane was incubated at $37^{\circ} \mathrm{C}$ for 1 hour with the horseradish peroxidase-labeled goat anti-rabbit IgG antibody (dilution 1:2000; ab6721). All antibodies above were obtained from Abcam Inc. (Cambridge, MA). Afterward, protein was visualized in enhanced chemiluminescence reaction solution (Pierce, Rockford, IL). With GAPDH adopted as the internal reference, the relative protein expression was presented as the ratio of the gray value of the target band/the band of GAPDH. ${ }^{18}$

\section{Luciferase Activity Assay}

The miRNAs that were modulated by LINC01133 were predicted on the bioinformatics website and verified with the use of dual-luciferase reporter assay. Shanghai Sangon Biotechnology Co, Ltd was commissioned to design and synthesize the LINC01133 wild-type (WT) and mutant-type (MUT) primers. Total RNA was extracted from cells, and WT and MUT primers were respectively used to amplify LINC01133. Next, the fragment of LINC01133 was inserted into the pGL3-Bashc luciferase reporter vector, named as LINC01133-WT-Luc; and the mutagenesis was performed in the binding region of LINC01133 and miR-205, and the mutant plasmid LINC01133-Mut-Luc was generated. LINC01133-WT-Luc and LINC01133-Mut-Luc were cotransfected into HEK-239T cells with miR-205 mimic and pRL-TK on the basis of the protocols provided on the Lipofectamine 2000. After a 6-hour transfection, with the solution substituted, the cells were harvested after 24 hours. The luciferase activity was determined using the DualLuciferase Reporter Assay Kit (Promega, Madison, WI) on the microplate reader at $560 \mathrm{~nm}$.

The bioinformatics website was employed for the prediction of the target gene of miR-205, and this target relationship between miR-205 and LRRK2 was confirmed with the use of the dual-luciferase reporter assay. The plasmids of LRRK2-WT-Luc and LRRK2-Mut-Luc, obtained from Shanghai Genechem Co, Ltd (Shanghai, China), were cotransfected into HEK-239T cells with miR-205 mimic and pRL-TK, followed by detection of the luciferase activity, as

\footnotetext{
Figure 2 Overexpression of LINC01133 inhibits proliferation, migration, and invasion of ovarian cancer cells. Ovarian cancer cell proliferation (A), colony formation (B), and cell migration and invasion (C and $\mathbf{D})$ were detected by ethynyl-2'-deoxyuridine (EdU; A), colony formation (B), and Transwell (C and $\mathbf{D})$ assays. Unpaired $t$-test was used for data analysis between two groups. One-way analysis of variance was used for comparisons among multiple groups with Tukey's post hoc test. The cell experiment was repeated three times. Measurement data are expressed as means \pm SD $(\mathbf{A}-\mathbf{D})$. ${ }^{*} P<0.05$ versus sh-LINC01133NC or LINC01133-NC. Scale bars $=50 \mu \mathrm{m}$ (A, C, and D).
} 

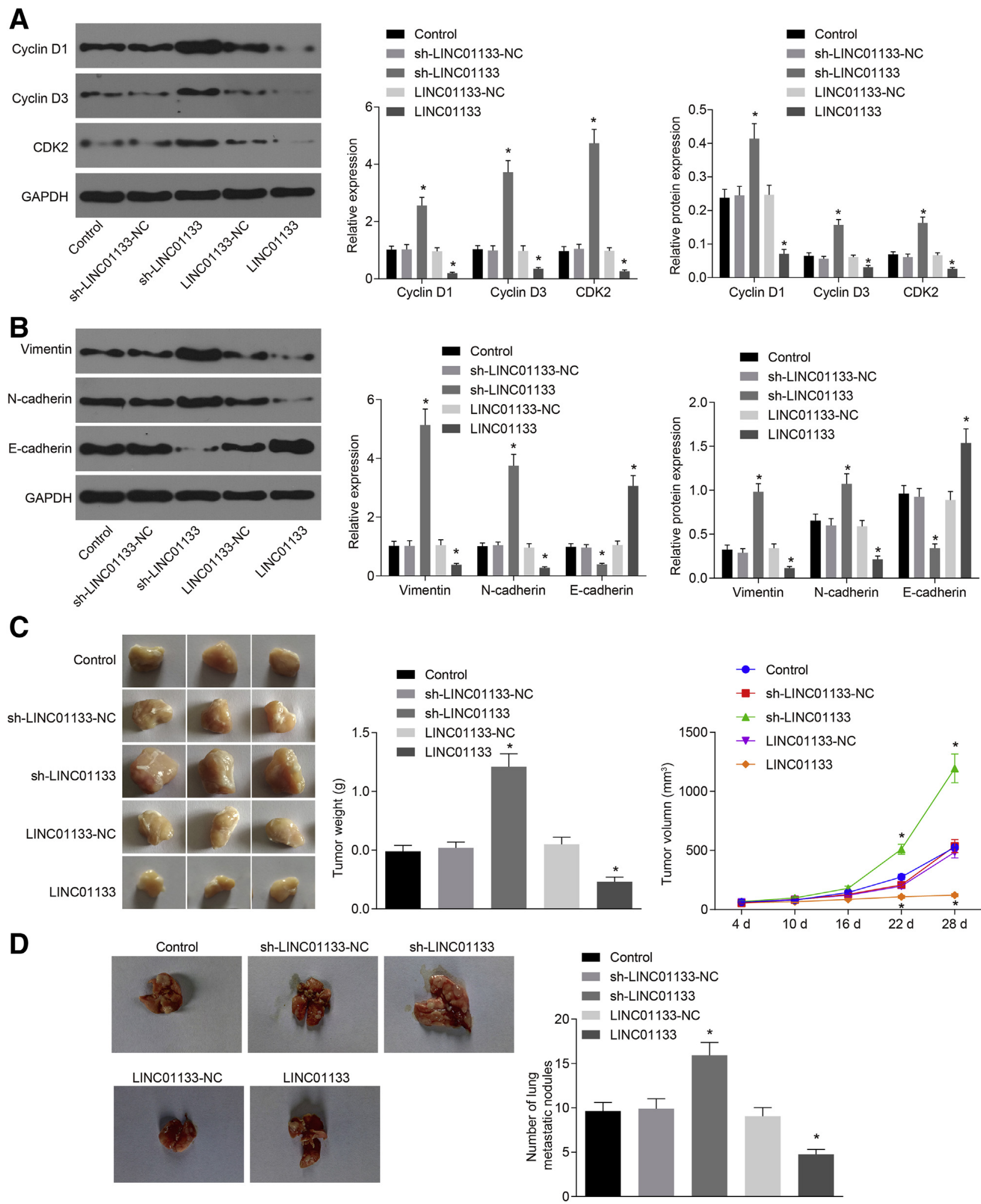

Figure 3 Overexpression of LINC01133 inhibits tumor formation and metastasis in vivo. Cyclin D1, cyclin D3, and cyclin-dependent kinase 2 (CDK2; A) and vimentin, $\mathrm{N}$-cadherin, and E-cadherin (B) levels in cells were determined by real-time quantitative RT-PCR and Western blot analysis. The tumorigenic (tumor weight and volume; C) and tumor metastasis (D) abilities were examined by the tumor formation (C) and in vivo metastasis (D) assays in nude mice. Unpaired $t$-test was used for data analysis between the two groups. One-way analysis of variance was used for comparisons among multiple groups. Repeated-measures analysis of variance was performed for analyzing the data at different time points with Tukey's post hoc test conducted. The cell experiment was repeated three times. Measurement data are expressed as means \pm SD (A-D). $n=10$ (C and D). ${ }^{*} P<0.05$ versus control. GAPDH, glyceraldehyde-3-phosphate dehydrogenase. 
mentioned above. The experiment was run in triplicates to obtain the mean value.

\section{RNA Pull Down}

The cells were transfected with $50 \mathrm{nmol} / \mathrm{L}$ biotin-labeled Bio-miR-205-probe and Bio-NC-probe (Wuhan GeneCreate Biological Engineering Co, Ltd, Wuhan, China) for 48 hours, followed by cell collection. Next, the cells were incubated for 10 minutes in Pierce immunoprecipitation lysis buffer (Thermo Fisher Scientific, Rockford, IL). The lysate was vortically incubated with Dynabeads streptavidin magnetic beads (Thermo Fisher Scientific) precoated with RNase-free bovine serum albumin and yeast tRNA (TRNABAK-RO; Sigma-Aldrich Chemical Company, St. Louis, MO) at $4^{\circ} \mathrm{C}$ overnight. Subsequently, the cells were rinsed twice with precooled lysis buffer, three times with low-salt buffer, and finally with high-salt buffer to obtain the magnetic separation of the RNA complex, with the supernatant harvested. The purification of miR205-combined RNA was performed using the TRIzol method, and RT-qPCR was employed to detect the enrichment of LINC01133. The experiment was performed in triplicate.

\section{RIP Data}

The cells were lysed using RNA immunoprecipitation (RIP) lysis buffer (P0013B; Beyotime Biotechnology Co, Ltd, Shanghai, China) for 5 minutes on an ice bath, followed by centrifugation at $16,873 \times g$ for 10 minutes at $4^{\circ} \mathrm{C}$, with the supernatant obtained. The binding of LINC01133 and argonaute 2 protein (Ago2) protein was detected using the RIP kit (Merck Millipore, Billerica, MA). A total of $50 \mu \mathrm{L}$ magnetic beads was resuspended in $100 \mu \mathrm{L}$ RIP wash buffer, which was then vertically incubated with $5 \mu \mathrm{g}$ rabbit anti-human Ago2 antibody (dilution 1:50; ab186733; Abcam Inc.) at $4^{\circ} \mathrm{C}$ for $>6$ hours. Subsequently, the magnetic bead-antibody complex was rinsed, followed by resuspension in $900 \mu \mathrm{L}$ RIP wash buffer and incubation overnight at $4^{\circ} \mathrm{C}$ with $100 \mu \mathrm{L}$ cellular supernatant. The sample was placed on the magnet base to collect the magnetic bead-protein complex, which was detached by proteinase $\mathrm{K}$ to extract RNA, followed by RT-qPCR. The untreated cellular supernatant was taken as the reference to calculate the binding percentage of LINC01133 to Ago2 protein, whereas SNRNP70 (Merck Millipore) was adopted as the positive control and rabbit anti-human $\operatorname{IgG}$ (dilution 1:100; ab109489; Abcam Inc.) was adopted as the negative control. The experiment was performed in triplicates.
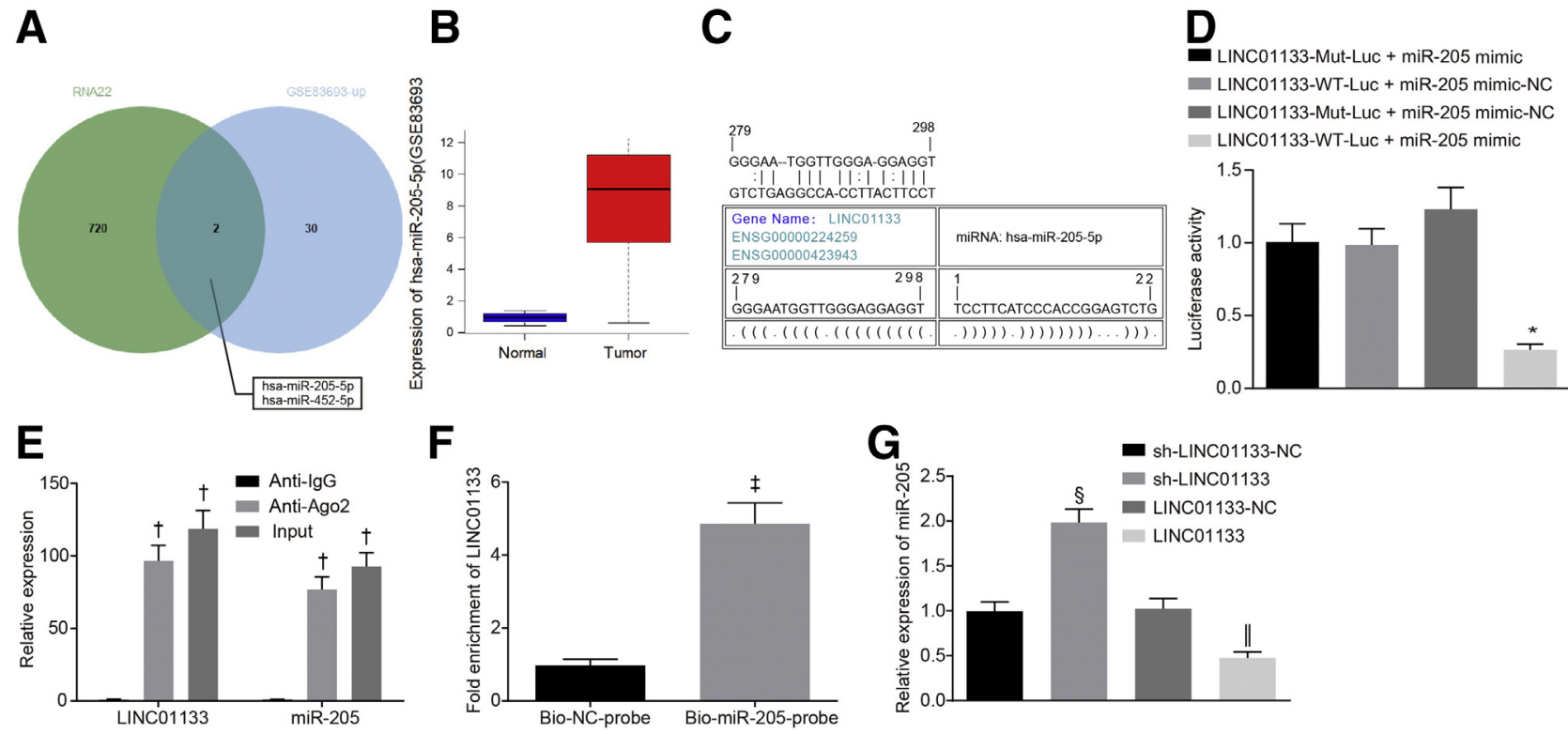

Figure 4 LINC01133 targets and binds to miR-205. A: miRNAs binding to LINC01133 were predicted by using RNA22. These miRNAs were compared with the miRNAs that were highly expressed in GSE83693. B: The expression of hsa-miR-452-5p in GSE83693 gene expression data set, where miR-205 was highly expressed in ovarian cancer relative to the normal group $(P=0.0041)$. C: The specific binding site between LINC01133 and miR-205. D: The targeting relationship between LINC01133 and miR-205, verified by dual-luciferase reporter assay. E: The targeting relationship between LINC01133 and miR-205 verified by RNA immunoprecipitation assay. F: The targeting relationship between LINC01133 and miR-205 testified by the RNA pull-down assay. G: The effect of LINC01133 on miR-205 expression determined by RT-PCR. Unpaired $t$-test was used for data analysis between the two groups. One-way analysis of variance was used for comparisons among multiple groups, with Tukey's post hoc test conducted. The cell experiment was repeated three times. Measurement data are expressed as means \pm SD $(\mathbf{D}-\mathbf{G}) .{ }^{*} P<0.05$ versus the LINC01133 + mutant (Mut) + luciferase (Luc) + miR-205 mimic, LINC01133 + wild type (WT) + LuC + miR-205 mimic-NC, and LINC01133 + Mut + LuC + miR-205 mimic-NC groups; ${ }^{\dagger} P<0.05$ versus sample pulled by IgG; ${ }^{\ddagger} P<0.05$ versus group labeled with Bio-NC probe; ${ }^{\S} P<0.05$ versus the sh-LINC01133-NC group; $\| P<0.05$ versus the LINC01133-NC group. 

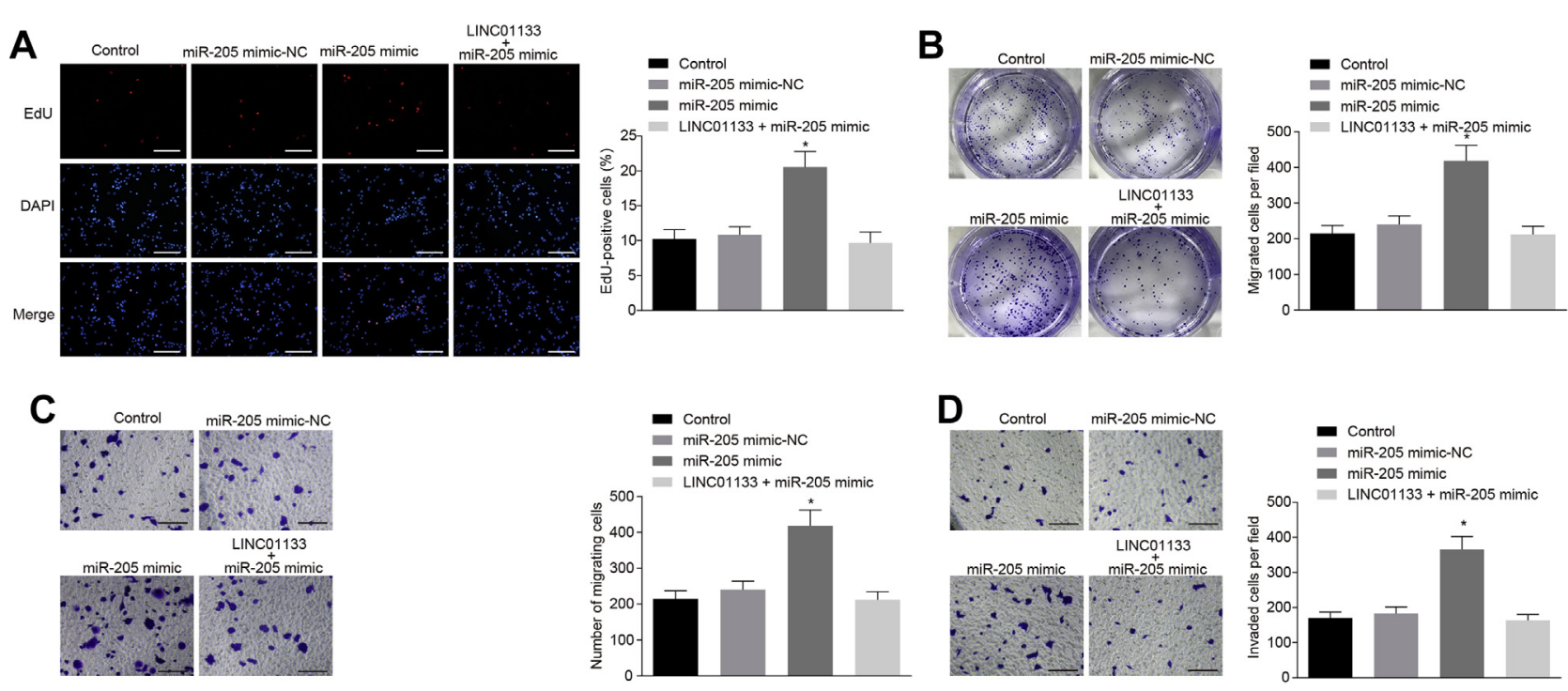

Figure 5 LINC01133 represses ovarian cancer cell proliferation, colony formation, invasion, and migration by targeting miR-205. Cell proliferation (A), colony formation (B), and cell migration and invasion (C and D) abilities of ovarian cancer were detected by ethynyl-2'-deoxyuridine (EdU; A), colony formation (B), and Transwell (C and $\mathbf{D}$ ) assays. Unpaired $t$-test was used for data analysis between the two groups. One-way analysis of variance was used for comparisons among multiple groups with Tukey's post hoc test performed. The experiment was repeated three times. Measurement data are expressed as means \pm SD (A-D). ${ }^{*} P<0.05$ versus the miR-205 mimic $-N C$ group. Scale bars $=50 \mu \mathrm{m}(\mathbf{A}, \mathbf{C}$, and $\mathbf{D})$.

\section{RNA Fluorescence in Situ Hybridization}

First, the cytoplasm and nucleus were separated. Next, the cells were treated with diethyl pyrocarbonate $-4 \%$ paraformaldehyde for 20-minute fixing at room temperature. Next, the cells were detached with proteinase K, followed by incubation with $0.1 \mathrm{~mol} / \mathrm{L}$ RNAse-free triethylamine for 10 minutes. Afterward, the slices were prehybridized for 1 hour with $200 \mu \mathrm{L}$ prehybridization solution at room temperature. The RNA probe was prepared using a prehybridization solution, followed by 5 -minute denaturation at $85^{\circ} \mathrm{C}$. Subsequently, the RNA probe was added for 14-hour incubation at $65^{\circ} \mathrm{C}$. Afterward, the slices were blocked for 1 hour at room temperature with buffer B2 and incubated with anti-digoxin antibody of alkaline phosphatase diluted with buffer $\mathrm{B} 2$ at the ratio of 5000:1 overnight at $4^{\circ} \mathrm{C}$. The slices were treated using buffer $\mathrm{B} 1$ washing at room temperature three times (20 minutes for each time), and buffer B3 balancing at room temperature twice, each session lasting for 5 to 10 minutes. The freshly prepared 5-bromo-4-chloro3 -indolyl phosphate/nitroblue tetrazolium coloring solution was added to visualize the slices for 3 to 24 hours with the avoidance of light exposure, after which the reaction was terminated with the addition of double-distilled water.

\section{Ethynyl-2'-Deoxyuridine Analysis}

The cells were plated in a 6-well plate at a density of $1 \times 10^{6}$ cells $/ \mathrm{mL}$ for 24 hours after transfection. Next, the cells were cultured with complete medium supplemented with ethynyl-2'-deoxyuridine for 2 to 3 days. The cells were fixed with $4 \%$ paraformaldehyde and incubated with glycine. Afterward, the cells were made permeable using
$0.5 \%$ Triton-X and incubated with the Apollo staining solution (Guangzhou RiboBio Co., Ltd., Guangzhou, China) and the Hoechst 33,342 reaction solution (Guangzhou RiboBio Co., Ltd.), avoiding exposure to light. The cells were observed and imaged under the inverted fluorescence microscope, followed by cell counting. The experiment was performed in triplicates to obtain the mean value.

\section{Colony Formation Assay}

After a 24-hour transfection, the cells from each group were detached with $0.25 \%$ trypsin and triturated into a single-cell suspension. Next, the cells were plated into a 6-well plate at a density of $1 \times 10^{4} / \mathrm{mL}$, followed by cell culture for 2 weeks. When the cell clones became visible to view with the naked eyes, the supernatant was discarded and the culture was terminated. The cells were fixed with $3.7 \%$ methanol for 10 minutes, followed by staining with $0.1 \%$ crystal violet for 10 to 30 minutes. Finally, the number of colonies with $>50$ cells was counted under the optical microscope using a low-power lens. The experiment was performed in triplicates to obtain the mean value.

\section{Transwell Assay}

After 24 hours of transfection, the cell suspension was added into the Transwell chamber, which was placed in a 24-well plate. Next, culture medium supplemented with $20 \%$ fetal bovine serum was added to the basolateral chamber for incubating cells for 24 to 48 hours. Afterward, with cells in the apical chamber wiped off, the cells were fixed in methanol for 20 minutes, stained with $0.1 \%$ crystal violet, and immersed in 33\% glacial acetic acid. Five high- 

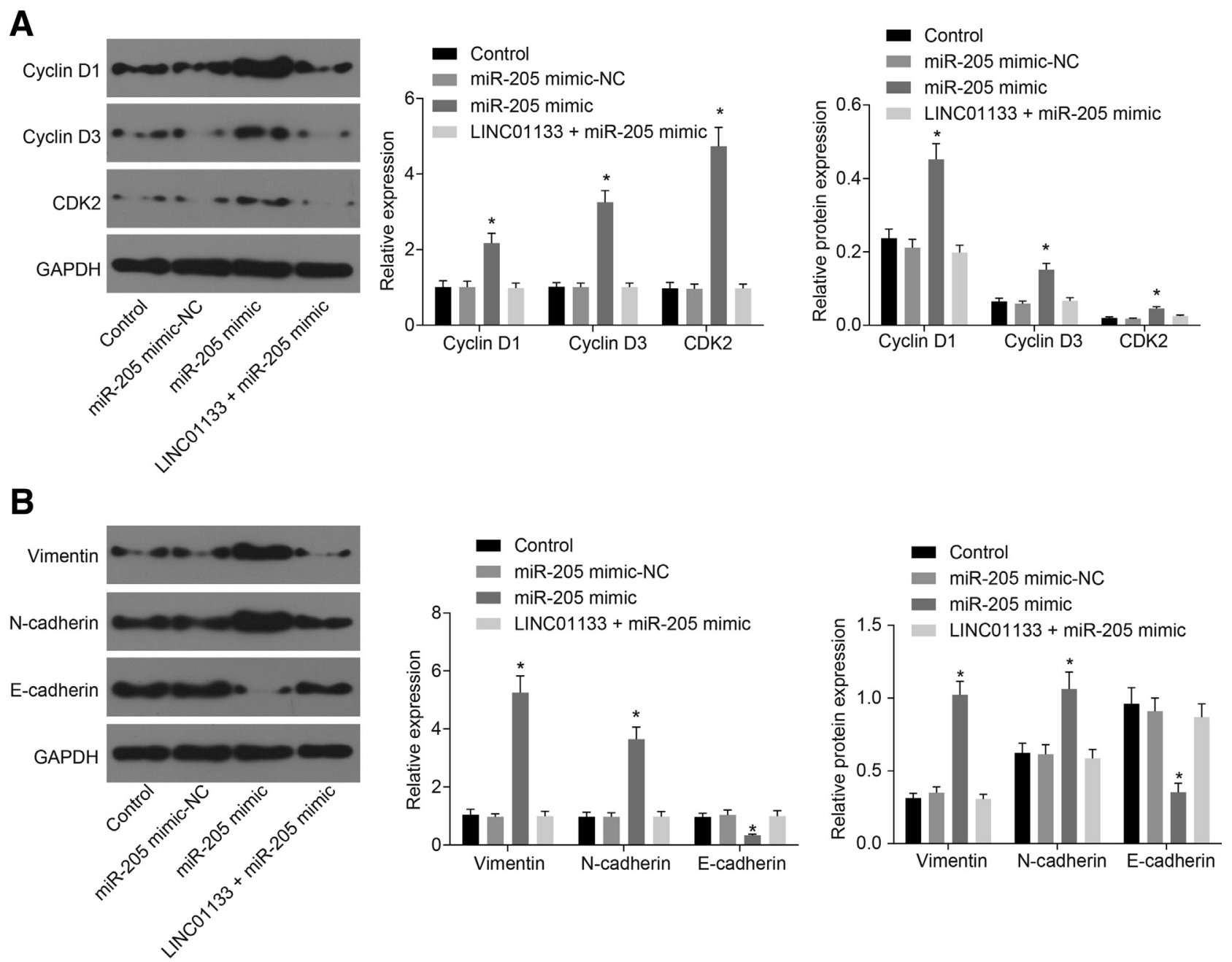

Figure 6 LINC01133 affects the expression of ovarian cancer cell proliferation-, invasion-, and migration-related genes by targeting miR-205. The mRNA and protein expression levels of cyclin D1, cyclin D3, and cyclin-dependent kinase 2 (CDK2; A) and vimentin, N-cadherin, and E-cadherin (B) were examined by real-time quantitative RT-PCR and Western blot analysis. Unpaired $t$-test was used for data analysis between two groups. One-way analysis of variance was used for comparisons among multiple groups with Tukey's post hoc test performed. The experiment was repeated three times. Measurement data are expressed as means \pm SD (A and $\mathbf{B})$. ${ }^{*} P<0.05$ versus the miR-205 mimic-NC group. GAPDH, glyceraldehyde-3-phosphate dehydrogenase.

power visual fields were selected under an optical microscope, and the number of migration/invasive cells was calculated. The procedure of invasion assay was the same as the one mentioned above, with additional coating of the apical chamber of Transwell with diluted Matrigel before the experiment. The experiment was performed in triplicates to obtain the mean value.

\section{Tumor Xenograft in Nude Mice}

Female BALB/c nude mice (aged 4 to 6 weeks; weighing 16 to $22 \mathrm{~g}$ ) were obtained from the Experimental Animal Center of Shandong University (Shandong, China) and randomly assigned into different groups, with 10 mice in each group. The mice had access to regular food and free access to water in a well-ventilated environment of $50 \%$ to $60 \%$ humidity and alternative light/darkness cycle at room temperature. The cells were detached 24 hours after transfection, followed by resuspension in serum-free medium
1640 and cell counting. Next, $1.5 \times 10^{6}$ cells were suspended in $0.1 \mathrm{~mL}$ serum-free Dulbecco's modified Eagle's medium, uniformly mixed with $0.1 \mathrm{~mL}$ extracellular matrix gel, and then subcutaneously injected into the back of nude mice. After 3 days, the same amounts of cells were injected into the nude mice at the same body part. After injection, tumor formation was checked after 2-day intervals, and the tumor volume was measured. After 4 weeks of tumor formation, nude mice were euthanized, and tumor specimens were removed, followed by measurement of the tumor weight and volume.

The volume of tumor was calculated as follows: $\left(\mathrm{L} \times \mathrm{W}^{2}\right) / 2$, where $\mathrm{L}$ indicated the length and $\mathrm{W}$ indicated the width of the tumor.

\section{Tumor Metastasis in Nude Mice}

Female BALB/c nude mice were randomly grouped with 10 mice in each group and fed as described above. After 24 
A

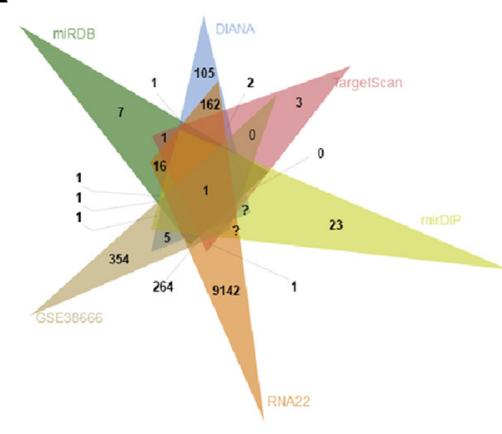

B

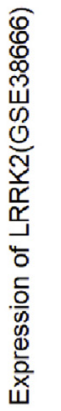

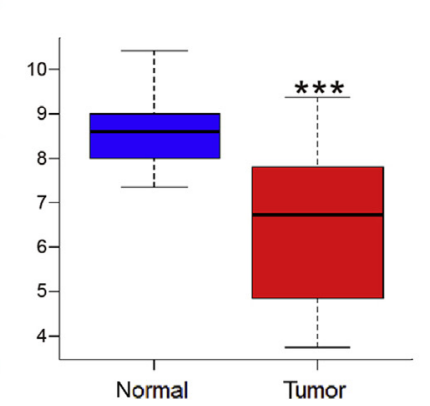

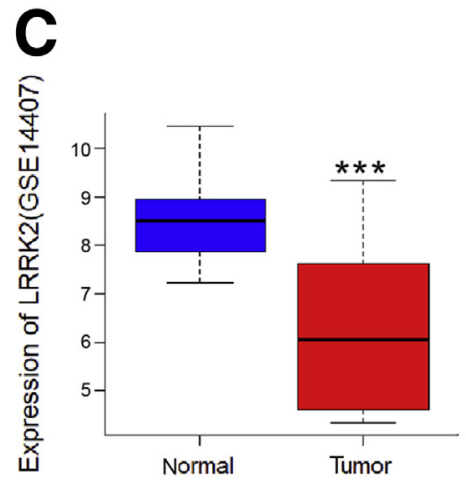

D

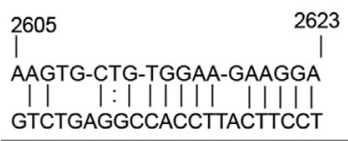

\begin{tabular}{|c|c|}
\hline $\begin{array}{l}\text { Gene Name: LRRK2 } \\
\text { ENSG00000188906 } \\
\text { ENSG00000298910 }\end{array}$ & miRNA: hsa-miR-205-5p \\
\hline $\begin{array}{l}2605 \\
\mid \\
\text { AAGTGCTGTGGAAGAAGGA }\end{array}$ & $\left.\right|_{\text {TCСТTCATCCCACCGGAGTCTG }} ^{22}$ \\
\hline 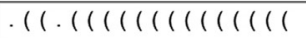 & 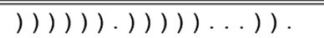 \\
\hline
\end{tabular}

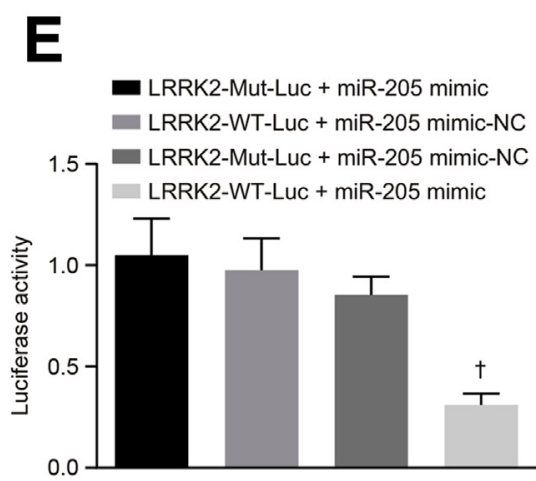

\section{E}

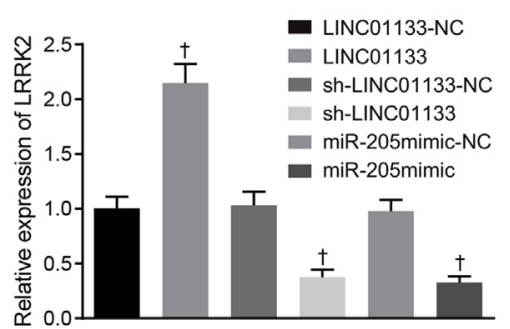

G

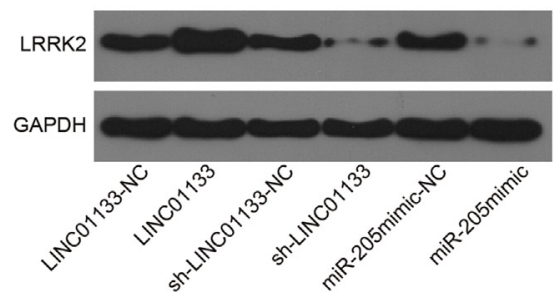

H

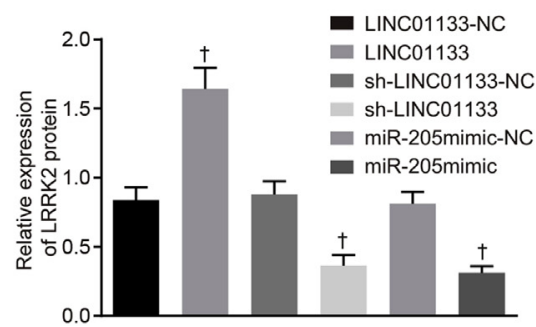

Figure 7 miR-205 targets and binds to LRRK2. A: The target genes of miR-205 were predicted by miRDB, DIANA, TargetScan, mirDIP, and RNA2; and the intersection genes were identified by comparing the genes with the differentially expressed genes in GSE38666. B: The expression of LRRK2 in the data set of GSE38666 in which LRRK2 expression is much lower in ovarian cancer tissues than that in normal tissues. C: The expression of LRRK2 in the data set of GSE14407 in which LRRK2 expression is significantly reduced in ovarian cancer tissues. D: The binding site between miR-205 and LRRK2, predicted by a bioinformatics website. E: The targeting relationship between miR-205 and $L R R K 2$, verified by dual-luciferase reporter gene assay. F: The mRNA expression of LRRK2 in cells detected by real-time quantitative RT-PCR. $\mathbf{G}$ and $\mathbf{H}$ : The protein expression of LRRK2, determined by Western blot analysis. One-way analysis of variance was used for comparisons among multiple groups with Tukey's post hoc test performed. Cell experiment was repeated three times. Measurement data are expressed as means \pm SD (E, F, and $\mathbf{H}) .{ }^{* *}{ }^{*} P<0.001$ versus normal; ${ }^{\dagger} P<0.05$ versus the LINC01133 NC/sh-LINC01133 NC/miR-205 mimic groups; GAPDH, glyceraldehyde-3-phosphate dehydrogenase.

hours of transfection, the cells were detached and resuspended in serum-free medium 1640 , with the cell concentration adjusted to $2 \times 10^{6}$ cells $/ \mathrm{mL}$. The nude mice were then injected with cell suspension into the tail vein using a skin test syringe ( $1 \mathrm{~mL}$ for each mouse). After 30 days of culture, the nude mice were euthanized, and the lung tissues were removed and adopted for the preparation of lung specimens by fixing with $10 \%$ neutral phosphate-buffered formalin. The number of metastatic tumors was counted.

\section{Statistical Analysis}

All statistical data were analyzed by SPSS software version 21.0 (IBM Corp., Armonk, NY). All data underwent normal distribution and a homogeneity of variance test. The measurement data conforming to the normal distribution were presented as means $\pm \mathrm{SD}$. The independent-sample $t$-test was applied to compare data between two groups. One-way analysis of variance was employed to compare data among 

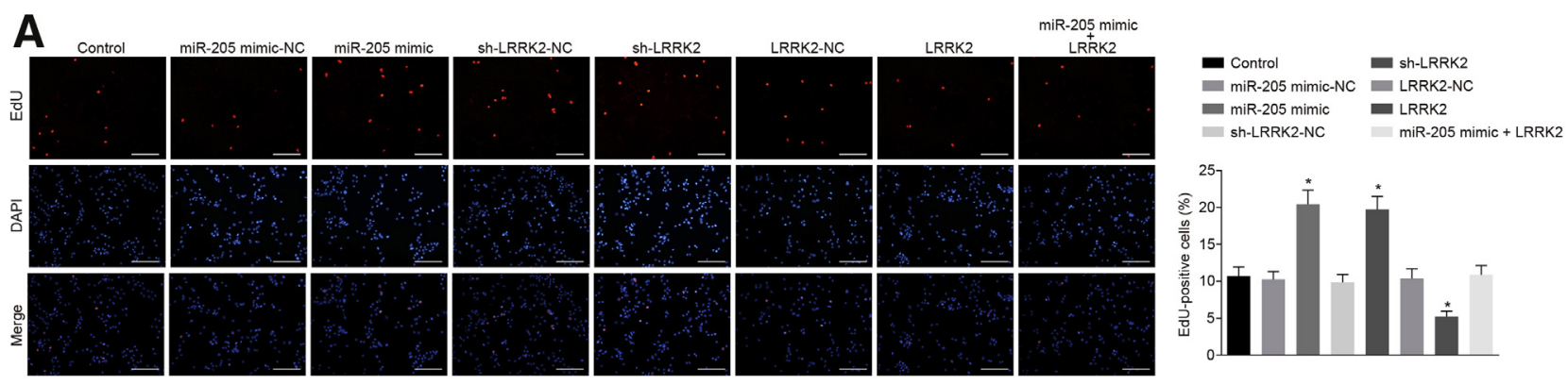

\section{B}
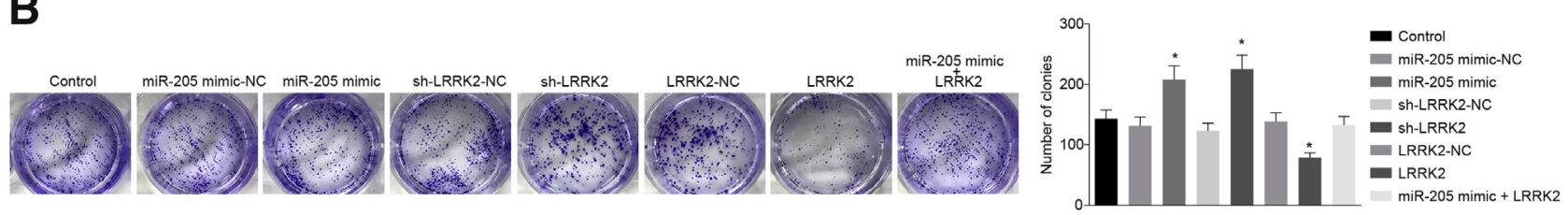

C
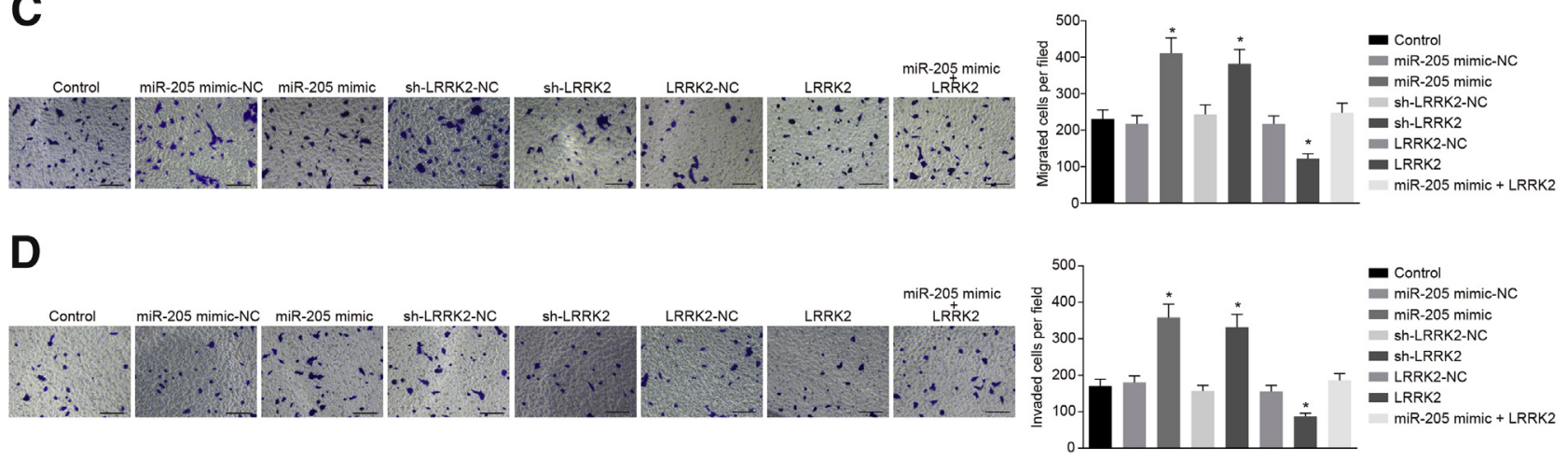

Figure 8 miR-205 enhances proliferation, colony number, migration, and invasion of ovarian cancer cells through targeting LRRK2. Ovarian cancer cell proliferation (A), clone formation (B), and migration and invasion (C and $\mathbf{D})$ abilities were determined by ethynyl-2'-deoxyuridine (EdU; A), colony formation (B), and Transwell (C and $\mathbf{D}$ ) assays. One-way analysis of variance was used for comparisons among multiple groups with Tukey's post hoc test conducted. Cell experiment was repeated three times. Measurement data are expressed as means \pm SD (A-D). ${ }^{*} P<0.05$ versus control. Scale bars $=50 \mu \mathrm{m}(\mathbf{A}, \mathbf{C}$, and $\mathbf{D})$.

multiple groups, and pairwise comparison was performed using post hoc test. The repeated measurement analysis of variance was adopted to analyze data at different time points, with Tukey's post hoc test. Statistical significance was set at $P<0.05$.

\section{Results}

\section{LINC01133 Is Poorly Expressed in Ovarian Cancer}

The differentially expressed genes of ovarian cancer were identified with the use of the software packages in R language from the gene expression data sets GSE14407 and GSE38666. The heat maps of the first 50 differentially expressed genes in the two data sets are illustrated in Figure 1, A and B. There was a decrease in the expression of LINC01133 in ovarian cancer tissues relative to the normal ovarian tissues. Therefore, LINC01133 was selected as an important molecule for subsequent analyses.

The expression of LINC01133 was lower in ovarian tissues than in normal ovarian tissues $(P<0.05)$ (Figure 1C). In addition, versus the normal ovarian epithelial cell line IOSE80, LINC01133 expression was diminished in three ovarian cancer epithelial cell lines (SKOV-3, HO-8910, and OVCAR-8); and the SKOV-3 cell line displayed the lowest expression of LINC01133 and, therefore, was selected for subsequent assays $(P<0.05)$ (Figure 1D). Analysis of The Cancer Genome Atlas data revealed that the ovarian cancer patients with high expression of LINC01133 exhibited longer overall survival than the ovarian cancer patients with low expression of LINC01133 $(P<0.05)$ (Figure 1E). The aforementioned results suggested that there might be a notable correlation between low expression of LINC01133 and the poor prognosis of ovarian cancer.

Moreover, on the basis of the prediction of IncATLAS, there was a high expression in LINC01133 in the cytoplasm (Figure 1F). Verification by RNA fluorescence in situ hybridization showed LINC01133 enrichment in the cytoplasm (Figure 1G).

\section{LINC01133 Suppresses Ovarian Cancer Cell Proliferation, Migration, and Invasion in Vitro and Tumor Formation and Metastasis in Vivo}

The underlying mechanisms were further studied. Relative to the normal cells, no notable difference was observed in 

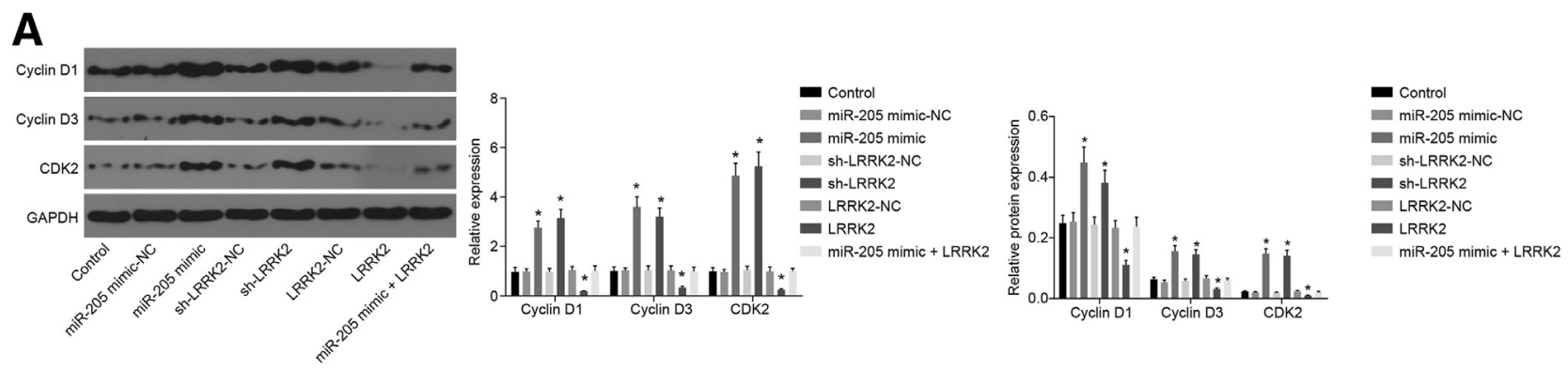

B
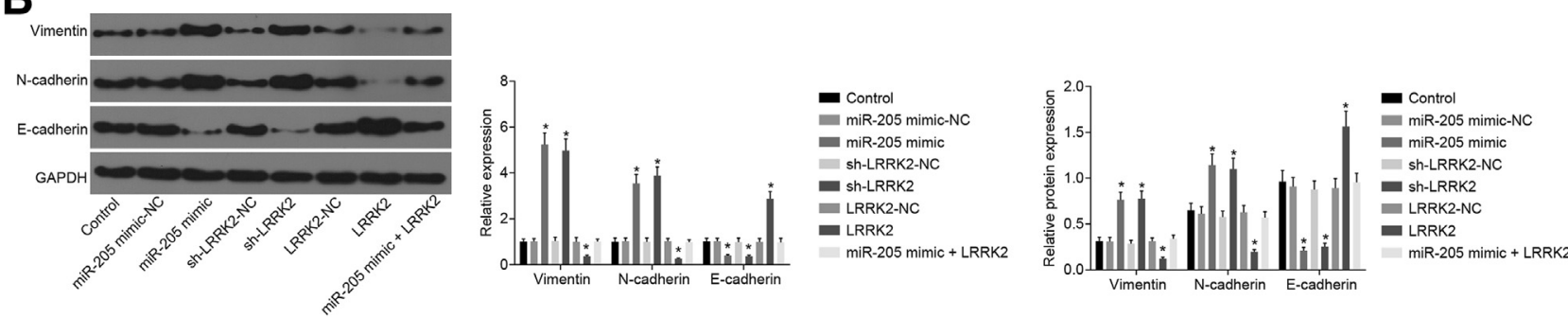

Figure 9 miR-205 affects the expression of ovarian cancer cell proliferation-, migration-, and invasion-related genes by targeting LRRK2. The mRNA and protein levels of cyclin D1, cyclin D3, and cyclin-dependent kinase 2 (CDK2; A) and vimentin, N-cadherin, and E-cadherin (B) in cells were examined by realtime quantitative RT-PCR and Western blot analysis. One-way analysis of variance was used for comparisons among multiple groups with Tukey's post hoc test conducted. Cell experiment was repeated three times. Measurement data are expressed as means \pm SD (A and $\mathbf{B})$. ${ }^{*} P<0.05$ versus control. GAPDH, glyceraldehyde-3-phosphate dehydrogenase.

cell proliferation, colony formation, invasion, and migration as well as the mRNA and protein expression of cyclin D1, cyclin D3, CDK2, vimentin, N-cadherin, and E-cadherin in response to transfection with sh-LINC01133-NC and LINC01133-NC $(P>0.05)$. In comparison with cells transfected with sh-LINC01133-NC or LINC01133-NC, the cells transfected with sh-LINC01133 presented with enhanced cell proliferation, colony formation, migration, and invasion, accompanied by an evident increase in expression of cyclin D1, cyclin D3, CDK2, vimentin, and $\mathrm{N}$-cadherin and diminished E-cadherin (all $P<0.05$ ). The cells transfected with LINC01133 displayed evidently reduced cell proliferation, colony formation, migration, and invasion abilities, accompanied with diminished expression of cyclin D1, cyclin D3, CDK2, vimentin, and N-cadherin, along with markedly increased E-cadherin $(P<0.05)$ (Figure 2 and Figure 3, A and B).

In contrast to the nude mice injected with normal cells, there was no obvious difference in the tumor weight and volume as well as the number of metastatic tumor nodules in the nude mice injected with sh-LINC01133-NC and LINC01133-NC $(P>0.05)$. In comparison with the nude mice injected with cells transfected with sh-LINC01133-NC or LINC01133-NC, the tumor weight and volume as well as the number of metastatic tumor nodules were elevated in the nude mice injected with shLINC01133, corresponding to a decrease in the nude mice injected with LINC01133 $(P<0.05)$. There was no notable difference in the above indicators in the nude mice bearing sh-LINC01133-NC-treated cells and the LINC01133NC-treated cells $(P>0.05)$ (Figure 3, C and D).
Therefore, LINC01133 overexpression resulted in the inhibition of proliferation, colony formation, migration, and invasion of ovarian cancer cells as well as the tumor formation and metastasis in vivo.

\section{LINC01133 Binds to miR-205 in Ovarian Cancer}

On the basis of the bioinformatic analysis, 69 differentially expressed miRNAs were screened out from the miRNA gene expression data set GSE83693, in which a high expression was found in 32 miRNAs. According to the prediction from RNA22, the binding site might exist between 722 miRNAs and LINC01133. The comparison between these miRNAs and 32 highly expressed miRNAs revealed that there were two miRNAs observed in both hsa-miR-205-5p and hsa-miR-452-5p (Figure 4A). Moreover, there was a high expression in miR-205 in ovarian cancer, which was identified from the data set of GSE83693 $(P=0.0041)$ (Figure 4B). Thus, miR-205 was selected as a differentially expressed miRNA for subsequent experiments.

Furthermore, the prediction from a bioinformatics website revealed that there existed a specific binding site between LINC01133 and miR-205 (Figure 4C). Thus, dual-luciferase reporter gene assay, RIP assay, and RNA pull down were conducted to verify whether LINC01133 targets miR-205 in cells. Versus the cells cotransfected with LINC01133-WTLuc and miR-205 mimic-NC, there was a remarkable decrease in the luciferase activity of cells cotransfected with LINC01133-WT-Luc and miR-205 mimic $(P<0.05)$, whereas there was no evident change in the luciferase activity after cotransfection with LINC01133-Mut-Luc and 


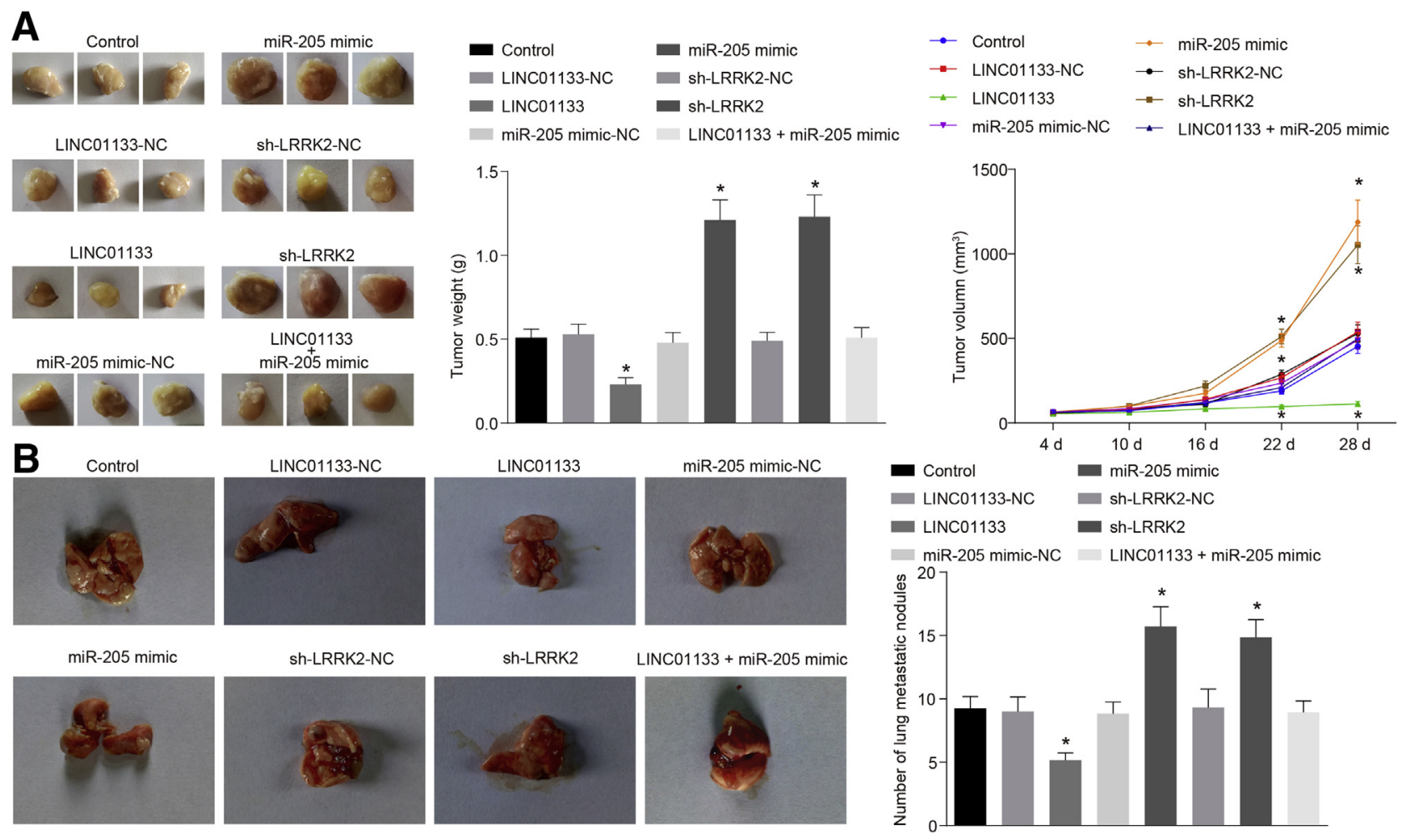

Figure 10 LINC01133 suppresses tumor formation and metastasis in vivo of ovarian cancer cells through elevation of LRRK2 by sponging miR-205. Tumorforming (A) and tumor metastasis (B) ability in vivo was determined by the xenograft tumor (A) and in vivo tumor metastasis (B) assay in nude mice. One-way analysis of variance was used for comparisons among multiple groups. Repeated-measures analysis of variance was performed at different time points with Tukey post hoc test performed, and the experiment was repeated three times. Measurement data are expressed as means \pm SD $(\mathbf{A}$ and $\mathbf{B}) . n=10(\mathbf{A}$ and $\mathbf{B})$. ${ }^{*} P<0.05$ versus control.

miR-205 mimic $(P>0.05)$ (Figure 4D). The RIP assay revealed that the expressions of LINC01133 and miR-205 were elevated in cells that received treatment with Ago2 than in cells treated with $\operatorname{IgG}(P<0.05)$ (Figure 4E). In addition, the results from the RNA pull down revealed that there was an up-regulation in LINC01133 in cells treated with miR-205 probe relative to cells treated with NC probe $(P<0.05)$ (Figure $4 \mathrm{~F}$ ). Moreover, the expression of miR205 was detected in cells treated with sh-LINC01133 or overexpressing LINC01133. Relative to the sh-LINC01133 $\mathrm{NC}$ treatment, the miR-205 expression was recovered after treatment of the sh-LINC01133, whereas it was suppressed after overexpressing LINC01133 (Figure 4G). The aforementioned findings were suggestive of the target relationship between LINC01133 and miR-205.

\section{LINC01133 Represses 0varian Cancer Cell Proliferation, Colony Formation, Invasion, and Migration by Binding to miR-205}

The influence of miR-205 was assessed on the proliferation, colony formation, invasion, and migration of ovarian cancer cells. The expression of proliferation-related (cyclin D1, cyclin D3, and CDK2), invasion-related, and migrationrelated (vimentin, $\mathrm{N}$-cadherin, and E-cadherin) factors was determined using ethynyl-2'-deoxyuridine assay, colony formation assay, Transwell assay, RT-qPCR, and Western blot analysis. Relative to the normal cells, there was no difference observed in the proliferation, colony formation, migration, and invasion as well as mRNA and protein expression of cyclin D1, cyclin D3, CDK2, vimentin, Ncadherin, and E-cadherin in the cells treated with miR-205 mimic-NC $(P>0.05)$. In comparison to cells treated with miR-205 mimic-NC, the cells treated with miR-205 mimic revealed evidently enhanced proliferation and cell colony formation ability; increased migration and invasion ability via pronounced elevation of cyclin D1, cyclin D3, CDK2, vimentin, and $\mathrm{N}$-cadherin expression; and notable decline of E-cadherin expression $(P<0.05)$. However, the above indicators did not differ in the cells treated with miR-205 mimic-NC and cotreated with LINC01133 and miR-205 mimic $(P>0.05)$ (Figures 5 and 6$)$.

Therefore, LINC01133 plays a role as an miR-205 sponge, suppressing proliferation, colony formation, invasion, and migration of ovarian cancer cells.

\section{miR-205 Targets LRRK2 in Ovarian Cancer}

The prediction of target genes of miR-205 was performed in miRDB, DIANA, TargetScan, mirDIP, and RNA22; and 

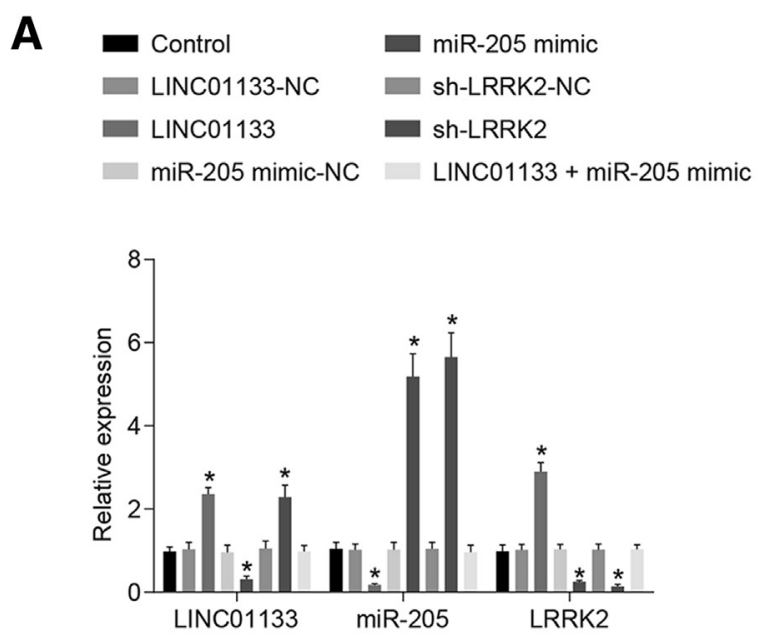

B
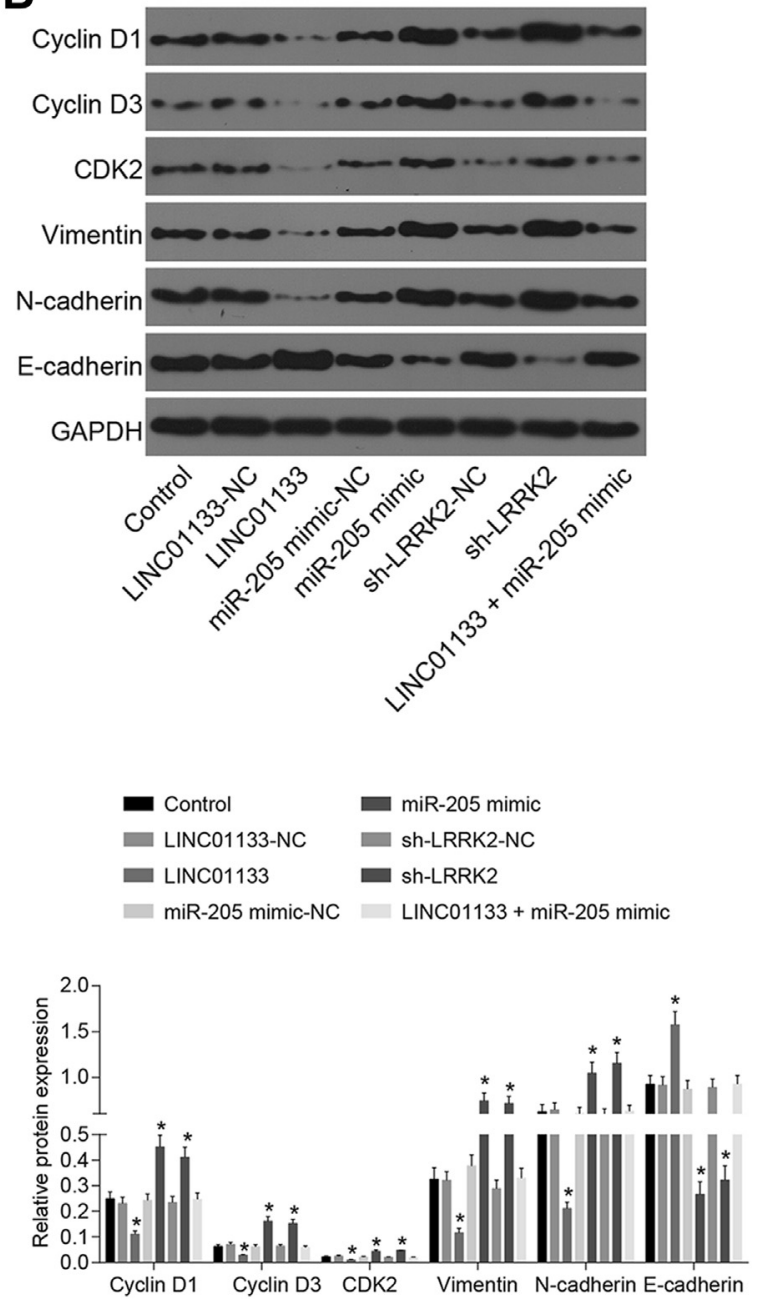

Figure 11 LINC01133 represses the expression of tumor formation- and metastasis-related genes in vivo through elevation of LRRK2 by sponging miR-205. The levels of LINC01133, miR-205, and LRRK2 (A) and cyclin D1, cyclin D3, cyclin-dependent kinase 2 (CDK2), vimentin, N-cadherin, and E-cadherin (B) determined by real-time quantitative RT-PCR (A) and Western blot (A and $\mathbf{B}$ ) analysis. One-way analysis of variance was used for comparisons among multiple groups. The experiment was repeated three times. Measurement data are expressed as means $\pm \mathrm{SD}(\mathbf{A}$ and $\mathbf{B}) .{ }^{*} P<0.05$ versus control. GAPDH, glyceraldehyde-3-phosphate dehydrogenase.
$87,383,38,171$, and 9708 target genes were identified according to target score $>80$ in miRDB, miTG score $>0.9$ in DIANA, cumulative weighted context ++ score $<-0.4$ in Targetscan, integrated score $>0.7$ in mirDIP, and prediction in RNA22, respectively. Besides, 639 and 678 differentially expressed genes were screened out from the data sets of GSE38666 and GSE14407, respectively. Only one intersection gene, LRRK2, was found between the differentially expressed genes in GSE38666 and the predicted target genes of miR-205 (Figure 7A). A lower expression of $L R R K 2$ was identified in ovarian cancer tissues versus that of the normal ovarian tissues in the data sets GSE38666 and GSE14407 (Figure 7, B and C). A prior study demonstrated that miR-205 negatively modulated LRRK. ${ }^{19}$ Thus, LRRK2 was explored as a target gene of miR-205.

The prediction of bioinformatics website revealed a binding site between miR-205 and LRRK2 (Figure 7D), and the dual-luciferase reporter assay was performed for further verification. Relative to the cells cotransfected with miR205 mimic-NC and LRRK2-WT-Luc, the luciferase activity of cells cotransfected with miR-205 mimic and LRRK2WT-Luc diminished $(P<0.05)$, whereas the luciferase activity was not different when cotransfected with LRRK2MUT-Luc $(P>0.05)$ (Figure 7E). To clarify how LINC01133 and miR-205 modulated their downstream gene $L R R K 2$, RT-qPCR and Western blot analysis were conducted to detect the $L R R K 2$ expression in cells treated with altered expression of LINC01133 or miR-205. The LRRK2 expression was increased in cells treated with overexpressing LINC01133, whereas a notable decline of LRRK2 expression was induced by sh-LINC01133 or by overexpressing miR-205 (Figure 7, F-H). Therefore, miR205 targets $L R R K 2$ in ovarian cancer.

miR-205 Accelerates Ovarian Cancer Cell Proliferation, Colony Formation, Migration, and Invasion by DownRegulating LRRK2

Relative to the normal cells, the cells treated with miR-205 mimic and sh-LRRK2 presented with an evident enhancement in proliferation and cell colony formation ability, and obviously increased migration and invasion ability via elevation of cyclin D1, cyclin D3, CDK2, vimentin, and Ncadherin expression and decline of E-cadherin expression (all $P<0.05$ ). The cells treated with LRRK2 exhibited an opposite trend (all $P<0.05$ ). In addition, after treatments with miR-205 mimic-NC, sh-LRRK2-NC, LRRK2-NC, and both miR-205 mimic and $L R R K 2$, cells displayed no pronounced change referring to the above mentioned markers $(P>0.05)$ (Figures 8 and 9).

In conclusion, the down-regulation of miR-205 repressed the cell proliferation, invasion, and migration of ovarian cancer by elevating LRRK2. 


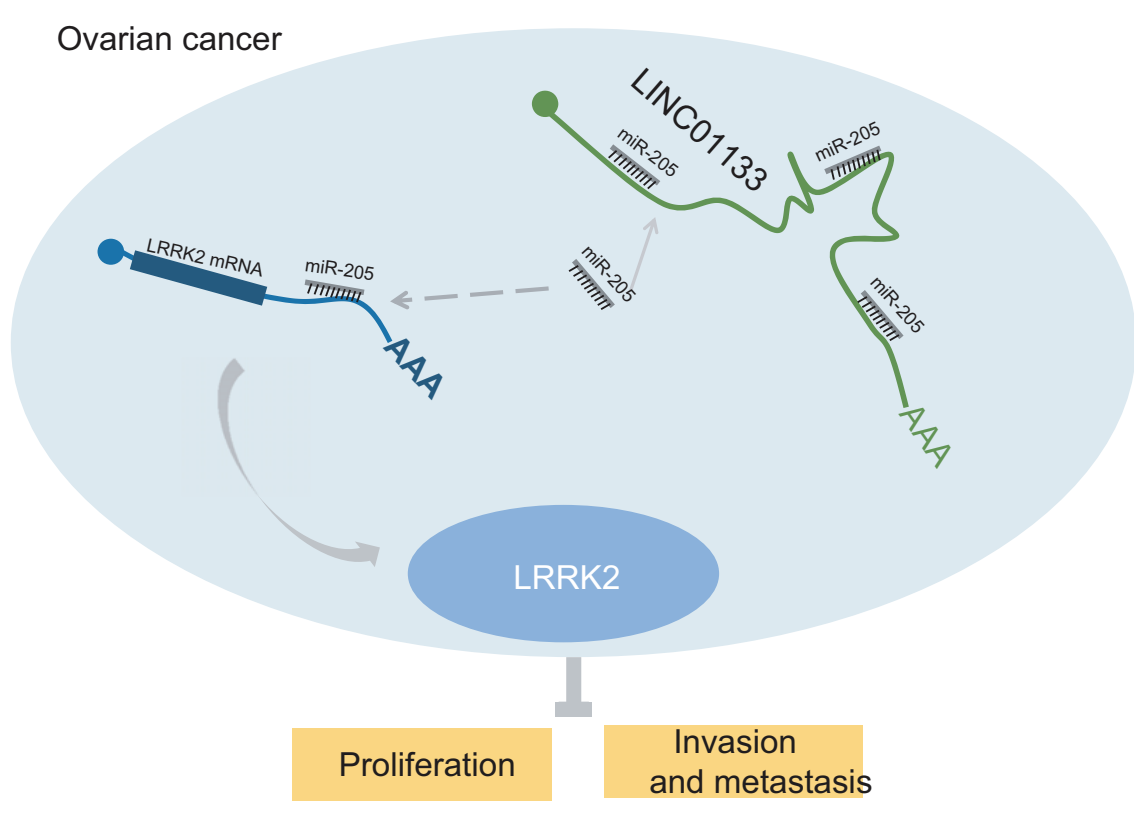

Figure 12 Schematic showing mechanism of LINC01133 in the progression of ovarian cancer. LINC01133 is expressed at low levels in the ovarian cancer. LINC01133 inhibits proliferation, migration, and invasion of ovarian cancer cells in vitro as well as tumor formation and metastasis in vivo by acting as a molecular sponge of miR-205 to up-regulate LRRK2.

\section{LINC01133 Elevates LRRK2 Expression as an miR-205 Sponge to Inhibit the Tumor Formation and Metastasis in Vivo}

Versus the nude mice injected with normal cells, the tumor weight and volume and the number of metastatic tumor nodules increased in the nude mice bearing the cells after treatment of miR-205 mimic and sh-LRRK2, whereas they markedly descended in the nude mice bearing the cells treated with LINC01133 (all $P<0.05$ ). There was no notable difference of these properties in the nude mice injected with cells after treatments of LINC01133-NC, miR205 mimic-NC, sh-LRRK2-NC, and cotreatment of LINC01133 and miR-205 mimic $(P>0.05)$ (Figure 10).

Subsequently, in contrast to the normal cells, the cells treated with miR-205 mimic displayed obviously increased expression of miR-205, whereas the expression of LINC01133 and LRRK2 diminished (all $P<0.05$ ), and the sh-LRRK2-treated cells exhibited pronounced decline of LRRK2 expression $(P<0.05)$. However, the LINC01133treated cells revealed an opposite result in contrast to the normal cells (all $P<0.05$ ). No notable difference in the expression of LINC01133, miR-205, and LRRK2 was found in the cells after treatments of LINC01133-NC, miR-205 mimic-NC, sh-LRRK2-NC, and cotreatment of LINC01133 and miR-205 mimic $(P>0.05)$ (Figure 11A).

Relative to the normal cells, there was a notable increase in the expression of cyclin D1, cyclin D3, CDK2, vimentin, and $\mathrm{N}$-cadherin, but that of E-cadherin was obviously reduced in the cells after treatment of miR-205 mimic and sh-LRRK2 (all $P<0.05$ ). However, the opposite trend was found in the LINC01133-treated cells $(P<0.05)$. There was no notable difference in the expression of the above indicators in the cells after treatments of LINC01133-NC,
miR-205 mimic-NC, sh-LRRK2-NC, and cotreatment of LINC01133 and miR-205 mimic $(P>0.05)$ (Figure 11B).

The aforementioned data revealed that LINC01133 elevation contributed to the suppression of tumor formation and metastasis in vivo of ovarian cancer by acting as an miR-205 sponge to up-regulate $L R R K 2$.

\section{Discussion}

The pivotal roles of lncRNAs that serve as competitive endogenous RNAs in the occurrence and development of ovarian cancer have been highlighted in recent evidence. ${ }^{20}$ The findings of this study suggested that LINC01133 acts as an miR-205 sponge to diminish cell proliferation, migration, and invasion of ovarian cancer by promoting LRRK2.

The data revealed that there was a reduction in the expression of LINC01133 and LRRK2 in ovarian cancer tissues, whereas miR-205 expression was elevated. LINC01133 is expressed at low levels in gastric cancer, and this down-regulation shares a positive correlation with the growth of gastric cancer cells. ${ }^{9}$ In line with our study, the high expression of miR-205 was proved to accelerate ovarian cancer cell invasion. ${ }^{21}$ LRRK2 was found to negatively regulate the production of innate inflammatory cytokines, and LRRK2 knockout mice presented with injured kidney autophagy and diminished expression of $L R R K 2$, which resulted in the invalid clearance of bacteria. ${ }^{22}$

Our research also revealed that LINC01133 could act as an miR-205 sponge, and LRRK2 was targeted by miR-205. In addition, LINC01133 acted as an miR-205 sponge, resulting in the elevation of LRRK2 expression, thereby restraining the cell proliferation, invasion, and migration of 
ovarian cancer, along with an impeded epithelialmesenchymal transition process. Cyclin D1 and cyclin D3 are poorly expressed in ovarian cancer cells after treatment with Notch inhibitor, which could induce apoptosis and suppress cell viability, migration, and angiogenesis. ${ }^{23}$ There is an overexpression of $C D K 2$ in primary ovarian cancers. ${ }^{24}$ There is a positive correlation between LINC01133 and Ecadherin, and a negative correlation with vimentin, and the overexpression of LINC01133 leads to suppressed tumor growth by down-regulating SRSF6 in colorectal cancer. ${ }^{10}$ Moreover, miR-205 accelerates cell proliferation and invasion of ovarian cancer via the inhibition of the expression of phosphatase and tensin homolog/mothers against decapentaplegic homolog $4 .^{12}$ Also, consistent with our findings, miR-205 negatively regulates $L R R K 2$ in Parkinson disease (PD). ${ }^{19}$ LRRK2 has been well established as one of the most commonly mutated genes in PD. ${ }^{25}$ LRRK2 levels are increased in the early stage of PD, ${ }^{26}$ and LRKK2 phosphorylation is closely associated with the abnormal motor behavior of levodopa-induced dyskinesia, which is crucial for PD treatment. ${ }^{27}$ Furthermore, LRRK2 modulates the balance between WNT/ $\beta$-catenin and WNT/planar cell polarity signaling, and this balance plays a crucial role in homeostasis of midbrain dopaminergic neurons, highlighting the notable role of LRRK2 in the pathogenesis of PD. ${ }^{28}$ In addition, nuclear factor of activated T cell levels are increased in the macrophage nucleus of knockout mice, which results in the up-regulation in the production of inflammatory cytokines and colitis symptoms. ${ }^{29}$ There is an ectopic expression of DGCR5 bound to miR-2861 and an evident suppression of cell growth and invasion in papillary thyroid carcinoma. ${ }^{30}$ In addition, the results from the tumor xenograft assay in nude mice revealed that overexpression of LINC01133 led to the suppression of the weight and volume of the tumor, along with a decrease in metastatic tumors.

In conclusion, LINC01133 is poorly expressed in ovarian cancer cells and tissues, whereas miR-205 is up-regulated. Moreover, LINC01133 results in the suppression of proliferation, invasion, and migration of ovarian cancer via the up-regulation of LRRK2 by sponging miR-205 (Figure 12). Therefore, LINC01133 can serve as a therapeutic biomarker in the treatment of ovarian cancer. Further studies are needed to examine the function of LINC01133 to broaden our understanding of the regulatory role of LINC01133 in cancer.

\section{Acknowledgments}

We thank the reviewers for helpful comments on this article.

\section{References}

1. Tew WP: Ovarian cancer in the older woman. J Geriatr Oncol 2016, 7: $354-361$
2. Siegel RL, Miller KD, Jemal A: Cancer statistics, 2018. CA Cancer J Clin 2018, 68:7-30

3. La Vecchia C: Ovarian cancer: epidemiology and risk factors. Eur J Cancer Prev 2017, 26:55-62

4. Erickson BK, Conner MG, Landen $\mathrm{CN}$ Jr: The role of the fallopian tube in the origin of ovarian cancer. Am J Obstet Gynecol 2013, 209: 409-414

5. Kim JY, Cho CH, Song HS: Targeted therapy of ovarian cancer including immune check point inhibitor. Korean J Intern Med 2017 , 32:798-804

6. Nikpayam E, Tasharrofi B, Sarrafzadeh S, Ghafouri-Fard S: The role of long non-coding RNAs in ovarian cancer. Iran Biomed J 2017, 21 : $3-15$

7. Kornienko AE, Guenzl PM, Barlow DP, Pauler FM: Gene regulation by the act of long non-coding RNA transcription. BMC Biol 2013 , 11:59

8. Zhang Y, Xu Y, Feng L, Li F, Sun Z, Wu T, Shi X, Li J, Li X: Comprehensive characterization of 1 cRNA-mRNA related ceRNA network across 12 major cancers. Oncotarget 2016, 7: 64148-64167

9. Yang XZ, Cheng TT, He QJ, Lei ZY, Chi J, Tang Z, Liao QX, Zhang H, Zeng LS, Cui SZ: LINC01133 as ceRNA inhibits gastric cancer progression by sponging miR-106a-3p to regulate APC expression and the Wnt/beta-catenin pathway. Mol Cancer 2018, $17: 126$

10. Kong J, Sun W, Li C, Wan L, Wang S, Wu Y, Xu E, Zhang H, Lai M: Long non-coding RNA LINC01133 inhibits epithelial-mesenchymal transition and metastasis in colorectal cancer by interacting with SRSF6. Cancer Lett 2016, 380:476-484

11. Kinose Y, Sawada K, Nakamura K, Kimura T: The role of microRNAs in ovarian cancer. Biomed Res Int 2014, 2014:249393

12. Chu $\mathrm{P}$, Liang A, Jiang A, Zong L: miR-205 regulates the proliferation and invasion of ovarian cancer cells via suppressing PTEN/SMAD4 expression. Oncol Lett 2018, 15:7571-7578

13. Han KA, Yoo L, Sung JY, Chung SA, Um JW, Kim H, Seol W, Chung KC: Leucine-rich repeat kinase 2 (LRRK2) stimulates IL-1betamediated inflammatory signaling through phosphorylation of RCAN1. Front Cell Neurosci 2017, 11:125

14. Committee for the Update of the Guide for the Care and Use of Laboratory Animals; National Research Council: Guide for the Care and Use of Laboratory Animals: Eighth Edition. Washington, DC, National Academies Press, 2011

15. Gautier L, Cope L, Bolstad BM, Irizarry RA: affy: Analysis of Affymetrix GeneChip data at the probe level. Bioinformatics 2004, 20: 307-315

16. Smyth GK: Linear models and empirical bayes methods for assessing differential expression in microarray experiments. Stat Appl Genet Mol Biol 2004, 3. Article3

17. Pecorelli S: Revised FIGO staging for carcinoma of the vulva, cervix, and endometrium. Int J Gynaecol Obstet 2009, 105:103-104

18. Rojo AI, Medina-Campos ON, Rada P, Zuniga-Toala A, LopezGazcon A, Espada S, Pedraza-Chaverri J, Cuadrado A: Signaling pathways activated by the phytochemical nordihydroguaiaretic acid contribute to a Keap1-independent regulation of Nrf2 stability: role of glycogen synthase kinase-3. Free Radic Biol Med 2012, 52: 473-487

19. Cho HJ, Liu G, Jin SM, Parisiadou L, Xie C, Yu J, Sun L, Ma B, Ding J, Vancraenenbroeck R, Lobbestael E, Baekelandt V, Taymans JM, He P, Troncoso JC, Shen Y, Cai H: MicroRNA-205 regulates the expression of Parkinson's disease-related leucine-rich repeat kinase 2 protein. Hum Mol Genet 2013, 22:608-620

20. Zhou M, Wang X, Shi H, Cheng L, Wang Z, Zhao H, Yang L, Sun J: Characterization of long non-coding RNA-associated ceRNA network to reveal potential prognostic lncRNA biomarkers in human ovarian cancer. Oncotarget 2016, 7:12598-12611

21. Wei J, Zhang L, Li J, Zhu S, Tai M, Mason CW, Chapman JA, Reynolds EA, Weiner CP, Zhou HH: MicroRNA-205 promotes cell 
invasion by repressing TCF21 in human ovarian cancer. J Ovarian Res 2017, 10:33

22. Dzamko NL: LRRK2 and the immune system. Adv Neurobiol 2017, $14: 123-143$

23. Kang H, Jeong JY, Song JY, Kim TH, Kim G, Huh JH, Kwon AY, Jung SG, An HJ: Notch3-specific inhibition using siRNA knockdown or GSI sensitizes paclitaxel-resistant ovarian cancer cells. Mol Carcinog 2016, 55:1196-1209

24. Marone M, Scambia G, Giannitelli C, Ferrandina G, Masciullo V, Bellacosa A, Benedetti-Panici P, Mancuso S: Analysis of cyclin E and CDK2 in ovarian cancer: gene amplification and RNA overexpression. Int J Cancer 1998, 75:34-39

25. Manzoni C: The LRRK2-macroautophagy axis and its relevance to Parkinson's disease. Biochem Soc Trans 2017, 45:155-162

26. Dzamko N, Gysbers AM, Bandopadhyay R, Bolliger MF, Uchino A, Zhao Y, Takao M, Wauters S, van de Berg WD, TakahashiFujigasaki J, Nichols RJ, Holton JL, Murayama S, Halliday GM:
LRRK2 levels and phosphorylation in Parkinson's disease brain and cases with restricted Lewy bodies. Mov Disord 2017, 32:423-432

27. Stanic J, Mellone M, Cirnaru MD, Perez-Carrion M, Zianni E, Di Luca M, Gardoni F, Piccoli G: LRRK2 phosphorylation level correlates with abnormal motor behaviour in an experimental model of levodopa-induced dyskinesias. Mol Brain 2016, 9:53

28. Salasova A, Yokota C, Potesil D, Zdrahal Z, Bryja V, Arenas E: A proteomic analysis of LRRK2 binding partners reveals interactions with multiple signaling components of the WNT/PCP pathway. Mol Neurodegener 2017, 12:54

29. Liu Z, Lee J, Krummey S, Lu W, Cai H, Lenardo MJ: The kinase LRRK2 is a regulator of the transcription factor NFAT that modulates the severity of inflammatory bowel disease. Nat Immunol 2011, 12: $1063-1070$

30. Chen F, Yin S, Zhu J, Liu P, Yang C, Feng Z, Deng Z: IncRNA DGCR5 acts as a tumor suppressor in papillary thyroid carcinoma via sequestering miR-2861. Exp Ther Med 2019, 17:895-900 